\title{
Virome comparisons in wild-diseased and healthy captive giant pandas
}

Wen Zhang ${ }^{1,2+}$, Shixing Yang ${ }^{1 \dagger}$, Tongling Shan ${ }^{3}$, Rong Hou', Zhijian Liu', Wang Li', Lianghua Guo ${ }^{5}$, Yan Wang ${ }^{1}$, Peng Chen ${ }^{2}$, Xiaochun Wang ${ }^{1}$, Feifei Feng ${ }^{2}$, Hua Wang ${ }^{1}$, Chao Chen², Quan Shen ${ }^{1}$, Chenglin Zhou ${ }^{4}$, Xiuguo Hua ${ }^{5}$, Li Cui ${ }^{5}$, Xutao Deng ${ }^{6}$, Zhihe Zhang ${ }^{2}$, Dunwu Qi ${ }^{2,7^{*}}$ and Eric Delwart ${ }^{6}$

\begin{abstract}
Background: The giant panda (Ailuropoda melanoleuca) is a vulnerable mammal herbivore living wild in central China. Viral infections have become a potential threat to the health of these endangered animals, but limited information related to these infections is available.
\end{abstract}

Methods: Using a viral metagenomic approach, we surveyed viruses in the feces, nasopharyngeal secretions, blood, and different tissues from a wild giant panda that died from an unknown disease, a healthy wild giant panda, and 46 healthy captive animals.

Results: The previously uncharacterized complete or near complete genomes of four viruses from three genera in Papillomaviridae family, six viruses in a proposed new Picornaviridae genus (Aimelvirus), two unclassified viruses related to posaviruses in Picornavirales order, 19 anelloviruses in four different clades of Anelloviridae family, four putative circoviruses, and 15 viruses belonging to the recently described Genomoviridae family were sequenced. Reflecting the diet of giant pandas, numerous insect virus sequences related to the families Iflaviridae, Dicistroviridae, Iridoviridae, Baculoviridae, Polydnaviridae, and subfamily Densovirinae and plant viruses sequences related to the families Tombusviridae, Partitiviridae, Secoviridae, Geminiviridae, Luteoviridae, Virgaviridae, and Rhabdoviridae; genus Umbravirus, Alphaflexiviridae, and Phycodnaviridae were also detected in fecal samples. A small number of insect virus sequences were also detected in the nasopharyngeal secretions of healthy giant pandas and lung tissues from the dead wild giant panda. Although the viral families present in the sick giant panda were also detected in the healthy ones, a higher proportion of papillomaviruses, picornaviruses, and anelloviruses reads were detected in the diseased panda.

Conclusion: This viral survey increases our understanding of eukaryotic viruses in giant pandas and provides a baseline for comparison to viruses detected in future infectious disease outbreaks. The similar viral families detected in sick and healthy giant pandas indicate that these viruses result in commensal infections in most immuno-competent animals.

Keywords: Giant panda, Viral metagenomics, Virome, Papillomavirus, Picornavirus, Anellovirus, Gemycircularvirus, Putative circovirus

\footnotetext{
* Correspondence: qidunwu@163.com

${ }^{\dagger}$ Equal contributors

${ }^{2}$ Sichuan Key Laboratory of Conservation Biology for Endangered Wildlife, Chengdu Research Base of Giant Panda Breeding, Chengdu, Sichuan 610081, China

${ }^{7}$ Sichuan Key Laboratory of Conservation Biology for Endangered Wildlife, Chengdu Research Base of Giant Panda Breeding, Chengdu, Sichuan 610000, China

Full list of author information is available at the end of the article
} the Creative Commons license, and indicate if changes were made. The Creative Commons Public Domain Dedication waiver (http://creativecommons.org/publicdomain/zero/1.0/) applies to the data made available in this article, unless otherwise stated. 


\section{Background}

The giant panda (Ailuropoda melanoleuca) is the sole strictly herbivorous bear species [1] within Ursidae family [2], whose other members are carnivores or omnivores. The State Forestry Administration of China reported 1864 wild giant pandas in the fourth national survey, representing a $16.8 \%$ increase over the previous decade resulting in its re-categorization as vulnerable in the International Union for Conservation of Nature's Red List of Threatened Species. Wild panda habitat and protected areas have significantly expanded in the last decade to 5.94 million hectares; however, anthropogenic habitat loss (e.g., deforestation) and poaching still threaten this vulnerable species [3]. The current global population of captive giant pandas is 425 , approaching the population development goal of 500 .

A low reproductive success and infectious diseases have hampered the development of captive and wild populations of the giant panda. Changes in the population or habitat of giant pandas may place animals at increased risk of infectious disease and hinder conservation efforts. The future of the endangered giant pandas depends in part on the development of protective measures against infectious diseases, especially viral infection. Although multiple studies of the bacterial content of giant panda feces have been reported [4-8], virologic study of giant panda has been limited to the identification and pathogenicity of single viruses including canine distemper virus, canine parvovirus, and influenza H1N1 [9-11].

Viral metagenomics has enabled the discovery of viral pathogens, viruses of unknown pathogenicity, and viruses of unknown cellular origins $[12,13]$. In this method, the concentration of host-derived and bacterial background nucleic acids in biological samples is reduced by filtration and nuclease digestion while viral nucleic acids are protected within capsids. Random amplification of DNA and RNA followed by deep sequencing generates metagenomics sequence information from which viral sequences are identified by translated sequence protein similarity searches to all known eukaryotic viral sequences.

\section{Methods}

\section{Sample collection and preparation}

In April 2015, a wild giant panda was found dying in Tangjiahe Nature Reserve in Guangyuan, Sichuan Province. The sick giant panda died after transportation to the nearest Dujiangyan giant panda first-aid center. This wild giant panda adult showed clinical signs including extreme emaciation (half of normal adult weight), extreme dehydration, anemia, mouth mucous membrane canker, and ascaris nematode worms. Serologic test indicated it was weakly positive for canine parvovirus antibody. In order to elucidate whether a viral infection was related to this animal's condition, feces, blood and nasopharyngeal secretion were collected prior to its death and heart, liver, spleen, lung, and kidney tissues collected after its death. In May 2015, another fresh fecal sample from an apparently healthy wild giant panda was collected from Tangjiahe Nature Reserve. Twenty-five fecal samples, 11 whole blood samples, and 10 nasopharyngeal secretion swabs were also collected from 46 clinically normal captive giant pandas in Chengdu Research Base of Giant Panda Breeding in Sichuan Province, China, from January 2014 to May 2015. All samples were collected by disposable materials and shipped on dry-ice. Fecal samples were re-suspended in ten volumes of phosphatebuffered saline (PBS) and vigorously vortexed for $5 \mathrm{~min}$. Fecal supernatants were then collected after centrifugation $(10 \mathrm{~min}, 15,000 \times g)$. The tips of respiratory swabs were immersed into $1 \mathrm{~mL}$ PBS and vigorously vortexed for $5 \mathrm{~min}$ and incubated for $30 \mathrm{~min}$ in $4{ }^{\circ} \mathrm{C}$. The supernatants were then collected after centrifugation $(10 \mathrm{~min}, 15,000 \times g)$. Tissue samples $(\sim 25 \mathrm{mg})$ were homogenized, frozen, and thawed three times on dryice, and the supernatants were then collected after centrifugation $(10 \mathrm{~min}, 15,000 \times g)$. The whole-blood samples were centrifuged $(10 \mathrm{~min}, 15,000 \times g)$ for the collection of plasma. Sample collection and all experiments in the present study were performed with the ethical approval given by the Ethics Committee of Jiangsu University and the reference number is No. UJS2014017.

\section{Viral metagenomic analysis}

Five hundred microliters of each supernatant was filtered through a $0.45-\mu \mathrm{m}$ filter (Millipore) to remove eukaryotic and bacterial cell-sized particles. The filtrates enriched in viral particles were treated with DNase and RNase to digest unprotected nucleic acid at $37{ }^{\circ} \mathrm{C}$ for $60 \mathrm{~min}$ [14-16]. Remaining total nucleic acid was then isolated using QiaAmp Mini Viral RNA kit (Qiagen) according to the manufacturer's protocol. Forty-one libraries were then constructed using Nextera XT DNA Sample Preparation Kit (Illumina) and sequenced using the MiSeq Illumina platform with 250 bases paired ends with dual barcoding for each pool. The information of each library is shown, where most of the libraries were constructed based on individual samples, except for 8 out of the 11 fecal libraries of the normal captive giant pandas consisting of 3 or 4 pooled fecal samples (Table 1). For bioinformatics analysis, paired-end reads of 250 bp generated by MiSeq were debarcoded using vendor software from Illumina. An in-house analysis pipeline running on a 32 -node Linux cluster was used to process the data. Reads were considered duplicates if bases 5 to 55 
Table 1 Library information of samples of giant pandas included in the present study

\begin{tabular}{|c|c|c|c|c|c|c|}
\hline Library ID & Sample type & No. of Sample & Healthy status & Habitation & Total no. of raw reads & No. of unique reads \\
\hline$\overline{\text { WB1 }}$ & Blood & 1 & Sick & Wild & 361,812 & 29,382 \\
\hline CB2 & Blood & 1 & Normal & Captive & 434,190 & 31,095 \\
\hline CB3 & Blood & 1 & Normal & Captive & 18,834 & 2852 \\
\hline CB4 & Blood & 1 & Normal & Captive & $2,527,742$ & 275,698 \\
\hline CB5 & Blood & 1 & Normal & Captive & 157,152 & 9274 \\
\hline CB6 & Blood & 1 & Normal & Captive & 428,670 & 29,353 \\
\hline CB7 & Blood & 1 & Normal & Captive & 11,236 & 1887 \\
\hline CB8 & Blood & 1 & Normal & Captive & 126,664 & 34,738 \\
\hline CB9 & Blood & 1 & Normal & Captive & $2,199,190$ & 156,333 \\
\hline CB10 & Blood & 1 & Normal & Captive & 491,388 & 38,996 \\
\hline CB11 & Blood & 1 & Normal & Captive & 227,730 & 26,883 \\
\hline CB12 & Blood & 1 & Normal & Captive & 176,224 & 21,045 \\
\hline 15FSW & Feces & 1 & Sick & Wild & 194,826 & 70,181 \\
\hline 16FNW & Feces & 1 & Normal & Wild & 528,634 & 164,066 \\
\hline $17 F N C$ & Feces & 3 & Normal & Captive & 977,118 & 534,051 \\
\hline $18 F N C$ & Feces & 3 & Normal & Captive & $1,137,802$ & 779,197 \\
\hline $19 F N C$ & Feces & 3 & Normal & Captive & 976,318 & 541,294 \\
\hline 20FNC & Feces & 3 & Normal & Captive & $1,608,664$ & $1,137,893$ \\
\hline $21 \mathrm{FNC}$ & Feces & 3 & Normal & Captive & $1,222,508$ & 933,678 \\
\hline $22 \mathrm{FNC}$ & Feces & 3 & Normal & Captive & $1,290,834$ & 906,394 \\
\hline $23 F N C$ & Feces & 3 & Normal & Captive & $2,906,028$ & $1,526,096$ \\
\hline $24 \mathrm{FNC}$ & Feces & 4 & Normal & Captive & 588,344 & 467,682 \\
\hline $25 F N C$ & Feces & 1 & Normal & Captive & 542,450 & 323,462 \\
\hline $26 F N C$ & Feces & 1 & Normal & Captive & 213,834 & 73,624 \\
\hline $27 F N C$ & Feces & 1 & Normal & Captive & 326,788 & 201,519 \\
\hline NSW1 & Oral swab & 1 & Sick & Wild & 292,802 & 120,260 \\
\hline NSC2 & Oral swab & 1 & Normal & Captive & 192,224 & 64,507 \\
\hline NSC3 & Oral swab & 1 & Normal & Captive & 321,150 & 99,124 \\
\hline NSC4 & Oral swab & 1 & Normal & Captive & 898,688 & 434,150 \\
\hline NSC5 & Oral swab & 1 & Normal & Captive & 208,236 & 53,208 \\
\hline NSC6 & Oral swab & 1 & Normal & Captive & 613,348 & 337,452 \\
\hline NSC7 & Oral swab & 1 & Normal & Captive & $1,266,946$ & 194,751 \\
\hline NSC8 & Oral swab & 1 & Normal & Captive & 235,500 & 62,890 \\
\hline NSC9 & Oral swab & 1 & Normal & Captive & 362,618 & 103,994 \\
\hline NSC10 & Oral swab & 1 & Normal & Captive & 319,506 & 88,356 \\
\hline NSC11 & Oral swab & 1 & Normal & Captive & 176,696 & 77,722 \\
\hline THE & Heart & 1 & Sick & Wild & 574,430 & 84,312 \\
\hline TLI & Liver & 1 & Sick & Wild & 546,204 & 109,832 \\
\hline TSP & Spleen & 1 & Sick & Wild & 447,030 & 35,613 \\
\hline TLU & Lung & 1 & Sick & Wild & 274,480 & 48,401 \\
\hline TKI & Kidney & 1 & Sick & Wild & 353,176 & 55,164 \\
\hline
\end{tabular}

were identical and only one random copy of duplicates was kept. Clonal reads were removed and low sequencing quality tails were trimmed using Phred quality score ten as the threshold. The unique read number of each library was shown in Table 1 . Adaptors were trimmed using the default parameters of VecScreen which is 
NCBI BLASTn with specialized parameters designed for adapter removal. The cleaned reads were de novo assembled within each barcode using the ENSEMBLE assembler [17]. Contigs and singlets reads are then matched against a customized viral proteome database using BLASTx with an $E$ value cutoff of $<10^{-5}$, where the virus BLASTx database was compiled using NCBI virus reference proteome (ftp://ftp.ncbi.nih.gov/refseq/ release/viral/) to which was added viral protein sequences from NCBI nr fasta file (based on annotation taxonomy in Virus Kingdom). Candidate viral hits are then compared to an in-house non-virus non-redundant (NVNR) protein database to remove false-positive viral hits, where the NVNR database was compiled using non-viral protein sequences extracted from NCBI nr fasta file (based on annotation taxonomy excluding Virus Kingdom). Contigs without significant BLASTx similarity to viral proteome database are searched against viral protein families in vFam database [18] using HMMER3 [19-21] to detect remote viral protein similarities. A web-based graphical user interface was developed to present users with the virus hits, along with taxonomy information and processing meta-information. The genome coverage of the target viruses were analyzed by Geneious (Biomatters).

\section{PCR confirmation and genome sequencing}

PCR confirmation was performed for the papillomavirus, gemycircularvirus, adenovirus, and insect viruses in the nasopharyngeal secretion samples; picornavirus, gyrovirus, and circoviruses in the fecal samples; gemycircularvirus in the blood samples; and anellovirus and the insect virus (Sacbrood virus) in the tissues. Inverse PCR were used to generate the complete genome of the novel anelloviruses in the blood samples and the gyrovirus in the fecal sample. PCR to bridge sequence gaps were used to acquire the complete genome of the picornaviruses in fecal samples and papillomavirus in nasopharyngeal secretion samples. Sequences and characteristics of the primers used in the present study are shown in Additional file 1: Table S1. Sanger method was used for sequencing of the PCR products.

\section{Quality control in the nucleic acid manipulation}

Standard precautions were used for all procedures to prevent the cross-sample contamination and nucleic acid degradation. Mainly, aerosol filter pipet tips were used to reduce the possibility of sample cross contamination, and all the materials (including microcentrifuge tubes, pipet tips) which directly contacted with nucleic acid samples were RNase and DNase free. The nucleic acid samples were dissolved in DEPC-treated water.

\section{Phylogenetic analysis}

Phylogenetic analyses were performed based on the predicted amino acid or nucleotide sequences in the present study, their closest viral relatives based on the BLASTx search in GenBank, and representative members of related viral species or genera. Sequence alignment was performed using CLUSTAL W with the default settings. Phylogenetic trees with 500 bootstrap resamples of the alignment data sets were generated using the Maximumlikelihood (ML) method in MEGA6.0. Bootstrap values (based on 500 replicates) for each node were given.

\section{Other sequence analysis}

Putative ORFs in the viral genome were predicted by Geneious 8.1 software or NCBI ORF finder. Putative exon and intron were predicted by Netgenes 2 at http://www.cbs.dtu.dk/services/NetGene2/. The conserved domains of the Pansaviruses (a novel virus in the order Picornavirales) from the feces of giant pandas were determined using the NCBI conserved domain search in combination with the Pfam conserved domain search $[22,23]$. Mapping raw data to the reference virus genome was performed using the low sensitivity/fastest parameter in Geneious software version 8.1, where the mapped reads show $>90 \%$ similarity to the reference genome.

\section{Nucleotide sequence accession numbers}

The novel viral genomes described in detail here were deposited in GenBank under the following accession numbers MF327529-MF327579. The raw sequence reads from the viral metagenomic libraries were deposited in the Short Read Archive of GenBank database under the accession number SRX2882233.

\section{Results}

\section{Overview of Virome}

Forty-one nucleic acid libraries from samples of giant pandas were generated and sequenced. The raw sequence reads numbers of each library generated by the Illumina MiSeq are shown in Table 1 . Raw sequence reads were binned by barcodes and quality-filtered, leaving highquality sequence reads which were de novo assembled within each barcode. The resulting sequence contigs and singlets were compared with the viral reference database and the GenBank non-redundant protein database using a BLASTx search with an E value cutoff of $<10^{-5}$. Translated sequences similar to those of known or suspected eukaryotic viral proteins are summarized in Table 2. In the fecal samples we detected eukaryotic viral sequences related to Picornaviridae, Genomoviridae, Circoviridae, Anelloviridae, Picobirnaviridae, other unclassified virus in picornavirales, and numerous insect and plant viruses. The eukaryotic viral sequences detected in blood samples included anellovirus genomes present in each of the 12 
Table 2 Eukaryotic viral sequences identified in the feces, blood, nasopharyngeal secretions, and tissues samples

\begin{tabular}{|c|c|c|c|c|c|c|c|c|c|c|}
\hline \multirow{2}{*}{$\begin{array}{l}\text { Library } \\
\text { ID }\end{array}$} & \multirow{2}{*}{$\begin{array}{l}\text { No. of } \\
\text { unique } \\
\text { reads }\end{array}$} & \multicolumn{9}{|c|}{ Reads no. of different viral species/families } \\
\hline & & Aenlloviridae & Adenoviridae & $\begin{array}{l}\text { Genomo/ } \\
\text { Circoviridae }\end{array}$ & Picrnaviridae & Papillomaviridae & $\begin{array}{l}\text { Posavirus- } \\
\text { like }\end{array}$ & $\begin{array}{l}\text { Picobirnavirus- } \\
\text { like }\end{array}$ & Plant virus & Insect virus \\
\hline WB1 & 29,382 & 3463 & 0 & 0 & 0 & 0 & 0 & 0 & 0 & 0 \\
\hline CB2 & 31,095 & 428 & 0 & 0 & 0 & 0 & 0 & 0 & 0 & 0 \\
\hline CB3 & 2852 & 61 & 0 & 0 & 0 & 0 & 0 & 0 & 0 & 0 \\
\hline CB4 & 275,698 & 530 & 0 & 6 & 0 & 0 & 0 & 0 & 0 & 0 \\
\hline CB5 & 9274 & 293 & 0 & 0 & 0 & 0 & 0 & 0 & 0 & 0 \\
\hline CB6 & 29,353 & 466 & 0 & 0 & 0 & 0 & 0 & 0 & 0 & 0 \\
\hline CB7 & 1887 & 26 & 0 & 0 & 0 & 0 & 0 & 0 & 0 & 0 \\
\hline CB8 & 34,738 & 10 & 0 & 0 & 0 & 0 & 0 & 0 & 0 & 0 \\
\hline CB9 & 156,333 & 4860 & 0 & 5 & 0 & 0 & 0 & 0 & 0 & 0 \\
\hline CB10 & 38,996 & 383 & 0 & 0 & 0 & 0 & 0 & 0 & 0 & 0 \\
\hline CB11 & 26,883 & 2411 & 0 & 0 & 0 & 0 & 0 & 0 & 0 & 0 \\
\hline CB12 & 21,045 & 584 & 0 & 0 & 0 & 0 & 0 & 0 & 0 & 0 \\
\hline 15FSW & 70,181 & 3 & 0 & 22 & 3309 & 0 & 74 & 0 & 15 & 70 \\
\hline 16FNW & 164,066 & 20 & 0 & 1258 & 1611 & 0 & 286 & 0 & 49 & 125 \\
\hline $17 F N C$ & 534,051 & 0 & 0 & 234 & 63 & 0 & 21 & 0 & 31 & 155 \\
\hline $18 F N C$ & 779,197 & 0 & 0 & 726 & 314 & 0 & 0 & 0 & 867 & 246 \\
\hline 19FNC & 541,294 & 0 & 0 & 926 & 177 & 0 & 0 & 0 & 602 & 86 \\
\hline 20FNC & $1,137,893$ & 0 & 0 & 771 & 170 & 0 & 0 & 0 & 259 & 1234 \\
\hline $21 F N C$ & 933,678 & 0 & 0 & 114 & 8 & 0 & 0 & 0 & 16 & 233 \\
\hline $22 \mathrm{FNC}$ & 906,394 & 8 & 0 & 2370 & 79 & 0 & 0 & 0 & 221 & 198 \\
\hline $23 F N C$ & 1,526,096 & 3 & 0 & 793 & 1382 & 0 & 0 & 24 & 2619 & 358 \\
\hline $24 F N C$ & 467,682 & 0 & 0 & 474 & 26 & 0 & 0 & 0 & 84 & 113 \\
\hline $25 F N C$ & 323,462 & 0 & 0 & 18 & 230 & 0 & 0 & 0 & 13 & 14 \\
\hline $26 F N C$ & 73,624 & 0 & 0 & 223 & 74 & 0 & 0 & 0 & 304 & 122 \\
\hline $27 F N C$ & 201,519 & 0 & 0 & 2196 & 21 & 0 & 0 & 0 & 14 & 35 \\
\hline NSW1 & 120,260 & 14 & 2 & 0 & 0 & 8507 & 0 & 0 & 0 & 0 \\
\hline NSC2 & 64,507 & 0 & 0 & 0 & 0 & 0 & 0 & 0 & 0 & 0 \\
\hline NSC3 & 99,124 & 0 & 0 & 11 & 0 & 0 & 0 & 0 & 0 & 0 \\
\hline NSC4 & 434,150 & 0 & 0 & 8 & 0 & 321 & 0 & 0 & 0 & 114 \\
\hline NSC5 & 53,208 & 0 & 0 & 0 & 0 & 0 & 0 & 0 & 0 & 0 \\
\hline NSC6 & 337,452 & 0 & 0 & 57 & 0 & 0 & 0 & 0 & 0 & 48 \\
\hline NSC7 & 194,751 & 0 & 0 & 0 & 0 & 0 & 0 & 0 & 0 & 0 \\
\hline NSC8 & 62,890 & 0 & 0 & 0 & 0 & 0 & 0 & 0 & 0 & 0 \\
\hline NSC9 & 103,994 & 0 & 0 & 15 & 0 & 0 & 0 & 0 & 0 & 29 \\
\hline NSC10 & 88,356 & 0 & 0 & 0 & 0 & 0 & 0 & 0 & 0 & 0 \\
\hline NSC11 & 77,722 & 4 & 15 & 0 & 0 & 261 & 0 & 0 & 0 & 0 \\
\hline THE & 84,312 & 5 & 0 & 0 & 0 & 0 & 0 & 0 & 0 & 0 \\
\hline TLI & 109,832 & 13 & 0 & 0 & 0 & 0 & 0 & 0 & 0 & 0 \\
\hline TSP & 35,613 & 56 & 0 & 0 & 0 & 0 & 0 & 0 & 0 & 0 \\
\hline TLU & 48,401 & 19 & 0 & 0 & 0 & 0 & 0 & 0 & 0 & 17 \\
\hline TKI & 55,164 & 0 & 0 & 0 & 0 & 0 & 0 & 0 & 0 & 0 \\
\hline
\end{tabular}


plasma derived libraries together with a small number of viral sequences showing similarity to gemycircularviruses in the newly described Genomoviridae family. In the nasopharyngeal secretion samples, we detected eukaryotic viral sequences related to Papillomaviridae, Anelloviridae, Genomoviridae, and Adenoviridae families and a low number of insect viral sequences. In four of the five tissues from the diseased animal, sequences related to Anelloviridae family were detected. A small number of insect viruses were also detected in the lung tissue. The overall composition of eukaryotic viruses detected in this study is represented in Fig. 1.

Comparison of the eukaryotic viruses in different giant pandas The composition of eukaryotic viruses of feces from the sick wild giant panda, the healthy wild giant panda, and three individually sequenced healthy captive giant pandas (25FNC, 26FNC, and 27FNC) is shown (Fig. 2a). All five samples were positive for picornaviruses, with the feces from the sick wild animal showing the highest percentage $(4.7 \%)$ relative to those of the other giant pandas (0.1-0.98\%) (Fig. 2a). Anellovirus was detected in all 12 plasma samples, with the sample from the sick wild giant panda showing the highest percentage of anellovirus sequences (11.8\%) while the percentage of anellovirus sequences in the other 11 samples ranged from 0.03 to $8.96 \%$ (Fig. $2 \mathrm{~b}$ ). The nasopharyngeal secretion sample of the sick wild giant panda contained abundant papillomavirus sequence reads whose sequence percentage $(7.07 \%)$ was higher than those of the only two of ten nasopharyngeal secretions from healthy captive animals that were positive for papillomavirus (with sequence percentages of 0.07 and $0.3 \%$, respectively) (Fig. 2c).

\section{Papillomaviruses in nasopharyngeal secretions of giant panda}

Papillomaviruses (PVs) are a highly diverse family of double-stranded circular DNA genomes approximately $8 \mathrm{~kb}$ in size known to infect a wide variety of mammals, as well as birds and reptiles. Some PV types cause benign or malignant epithelial tumors of the skin and mucous membranes in their hosts, while others are commonly detected on healthy human skin, as well as on that of different animals. Papillomaviruses are classified into genera based on the sequence of the highly conserved ORF L1 [24]. While the overwhelming majority of PVs only infect epithelium and are highly host specific [24, 25], the bovine papillomaviruses (BPVs) of the Deltapapillomavirus genus have the ability to infect both epithelial and mesenchymal cells and to infect multiple species [26]. Recently, more complete genomes of PVs were reported in non-human species, including one from the oral mucosa of a polar bear [27] the closest extant relative of giant panda.

Here, we characterized four complete PV genomes from the nasopharyngeal secretions of giant pandas $(A$. melanoleuca), hereafter referred to as AmPV1, AmPV2, AmPV3, and AmPV4. The genomes of the four AmPVs are described in Additional file 2: Table S2. AmPV1 and AmPV2 were both found in the nasopharyngeal

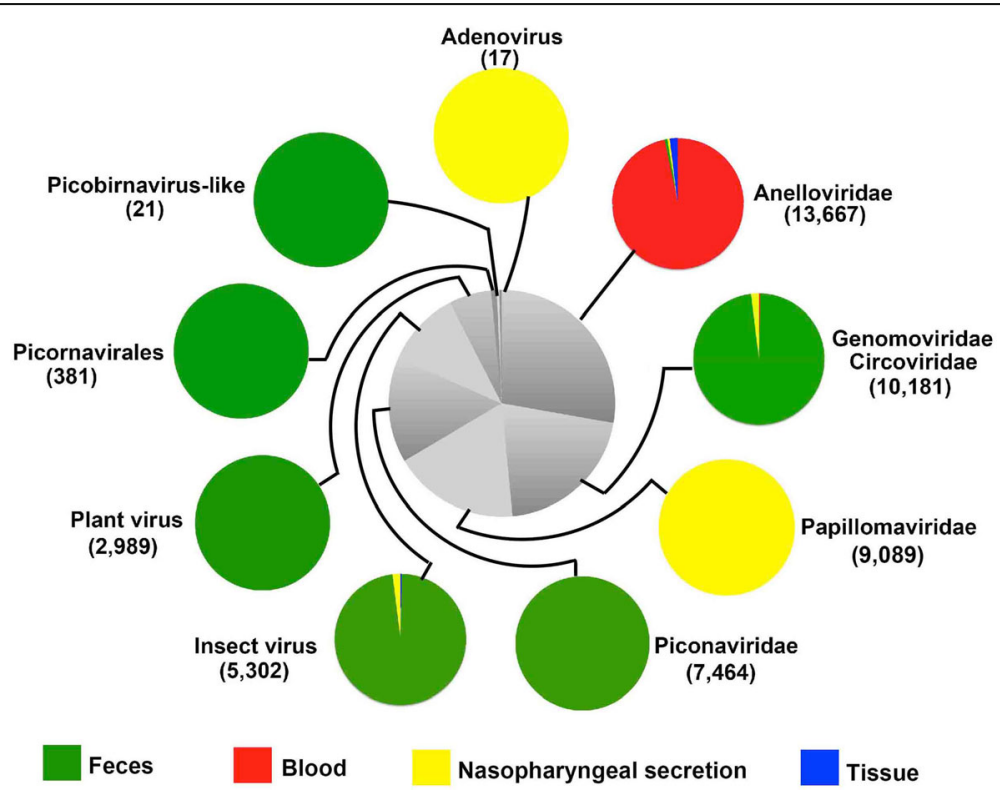

Fig. 1 The composition and sample distribution of eukaryotic viruses detected in giant pandas. The pie chart in the center showed the approximate percentages of the nine virus groups detected in all types of sample. The nine circumjacent smaller pie charts indicated the approximate percentage of virus sequence from different type samples. Sample types were showed in different colors 


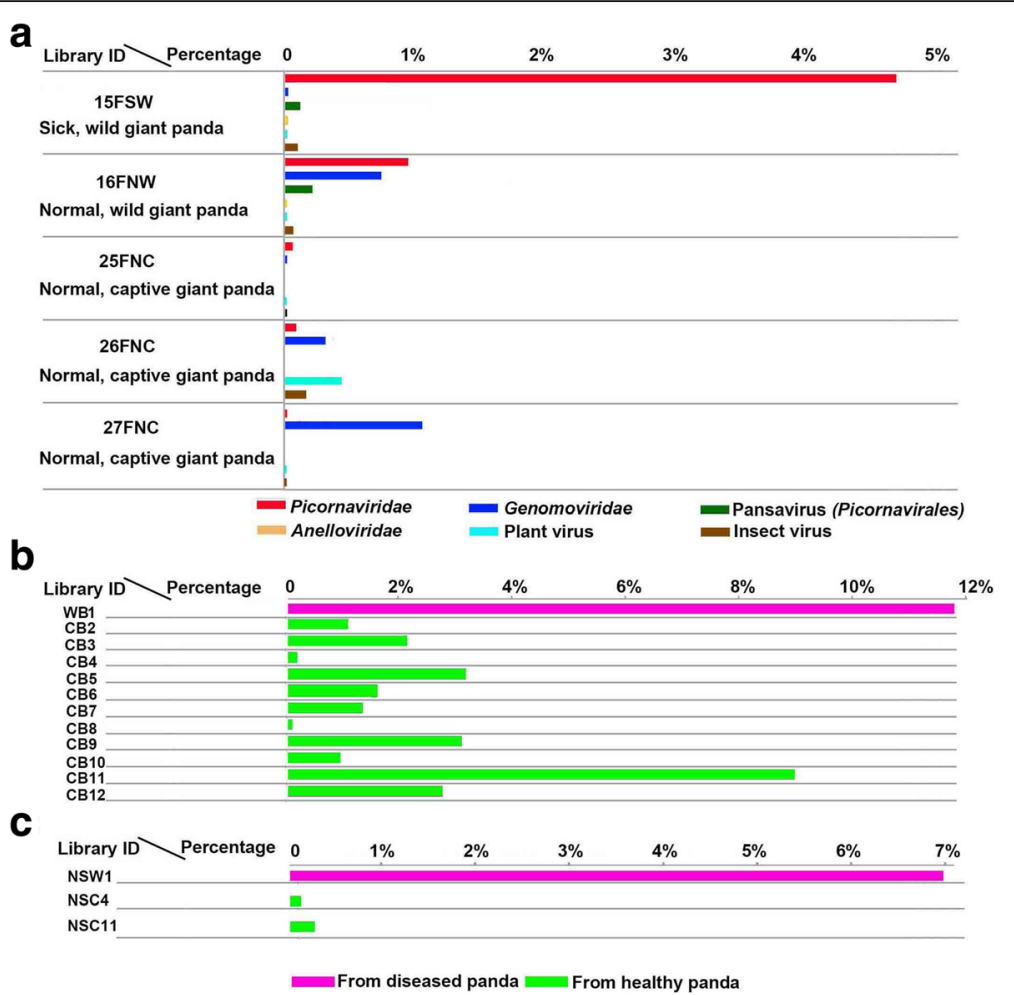

Fig. 2 Virome comparisons of different samples of giant pandas based on BLASTx to the GenBank non-redundant database $(E$ value of $<10-5)$. a Percentage of virus-like sequence reads with similarity to eukaryotic viruses in the five libraries constructing based on five individual fecal samples. Viruses belonging to different groups were marked with different colors. b Percentage of anellovirus-related sequences in blood samples of different individual animals, where the bar related to the wild giant panda was marked with green and those related to the captive ones were marked with purple. c Percentage of papillomavirus-related sequences in the positive nasopharyngeal secretion swabs, where the bar related to the diseased giant panda was marked with purple and those related to captive ones were marked with green

secretion of the diseased wild giant panda and shared $62.5 \%$ nucleotide sequence similarity over the complete genome. AmPV3 and AmPV4 were detected in the nasopharyngeal secretions from the two healthy captive giant pandas, respectively, and showed $<55 \%$ sequence similarities to each other and AmPV1 and AmPV2 over the complete genome based on pairwise comparison. Genome sizes of the four AmPVs were 7676, 7582, 7886, and 7996 bp, with GC contents of $43.5,45.6,58.6$, and 38.5\%, respectively. Five distinct ORFs on the same coding strand were identified in all four PV genomes, including the early genes E6, E1, and E2 and the late genes L2 and L1 (Fig. 3a). Analysis of the deduced amino acid sequences revealed that many of the classic PV-specific elements were present in the four AmPVs (Additional file 2: Table S2).

To determine the divergence in sequence between the AmPVs found here and other PVs, amino acid sequence alignment of the complete L1 protein was performed, and an ML phylogenetic tree was generated. The sequence alignment included the four AmPVs identified here, the best BLASTp matches in GenBank based on the L1 proteins, and 66 representative species from each of the currently recognized genera [28]. Phylogenetic analysis indicated that AmPV1 and AmPV2 fell within a separate clade also including UmPV identified in the oral mucosa of a polar bear [27] (Fig. 3b). AmPV1 had the L1 identities of 73.6 and $73.1 \%$ with those of AmPV2 and UmPV1, respectively, while AmPV2 had identity of 91.1\% with the L1 of UmPV1. AmPV3 clustered with ZcPV (from California sea lion), sharing $62.3 \%$ L1 protein sequence identity. AmPV4 clustered with PIPV, CPV1, and FcaPV1 from different carnivores, respectively, sharing L1 protein sequence identities of $71.4,68.3$, and $68.2 \%$.

According to the International Committee on Taxonomy of Viruses (ICTV), papillomaviruses are classified using the conserved ORF L1 nucleotide sequence with PVs within the same genus expected to have greater than approximately $60 \%$ nucleotide identity and PVs within the same species greater than $70 \%$ identity. AmPV1 and AmPV2 both have the closest relative of UmPV1 (GenBank no. NC_010739) in GenBank and cluster together within Omegapapillomavirus (Fig. 3b). Based on the ORF L1 nucleotide sequence, AmPV2 has similarity of $80.3 \%$ to UmPV1, while AmPV1 has similarities of 69.6 and $68.5 \%$ to AmPV2 and UmPV1, respectively. 

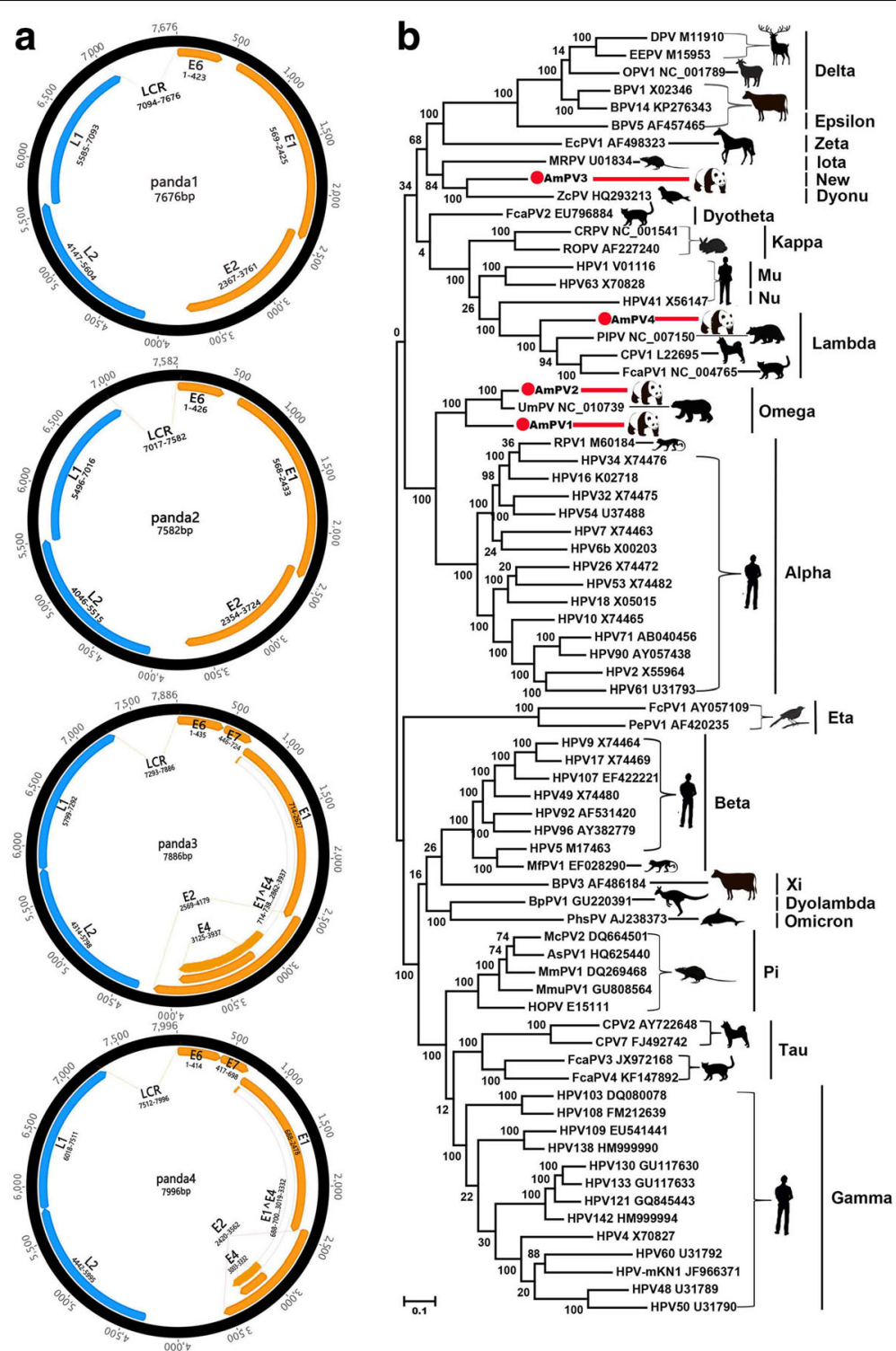

Fig. 3 Genomic organization and phylogenetic analysis of the Papillomaviruses identified in the giant pandas. a Genomic organization of the AmPVs identified in the nasopharyngeal secretions of giant pandas. $\mathbf{b}$ Phylogenetic analysis was performed based on the amino acid sequence of L1 protein. The sequence alignments included the four AmPVs identified here, the best BLASTp matches in GenBank based on the L1 proteins of the AmPV1-4, and 66 representative species from each of the currently recognized genera. Silhouettes of the hosts included in the phylogenetic analysis were showed on branches. Papillomaviruses identified in this study was labeled with red dots

Therefore, AmPV1 and AmPV2 together with UmPV1 belong to Omegapapillomavirus, where AmPV1 is a proposed new PV species while AmPV2 and UmPV1 belong to two different types within the same species. BLASTN search in GenBank with ORF L1 nucleotide sequence indicated that AmPV3 has the best match of $\mathrm{ZcPV}$ (GenBank no. HQ293213) and shares 58.3\% similarity with it, suggesting that AmPV3 belongs to a proposed new genus. Based on the ORF L1 nucleotide sequence, AmPV4 has the best BLASTN searching match with PIPV (GenBank no. NC_007150), sharing identity of
68.1\%, which suggests AmPV4 belongs to a new species within Lambdapapillomavirus genus.

\section{New picornavirus genus in feces of giant panda}

Picornaviruses are small, non-enveloped, positive-sense, single-stranded RNA viruses with a genome size of 7.1 to $8.9 \mathrm{~kb}$, encoding a single polyprotein. The family Picornaviridae belongs to the order Picornavirales and currently consists of at least 54 species grouped into 31 genera. Additionally, there are four proposed new genera (each containing a single proposed species) and 24 
proposed new species within existing genera (www.picornaviridae.com). Here, we characterized a novel picornavirus in fecal samples, which was provisionally named Aimelvirus (Ailuropoda melanoleuca virus). All libraries of fecal samples were positive for Aimelvirus. Six different complete genomes of Aimelvirus could be generated from six different fecal libraries, including two strains (Aimelvirus 1 and 6) from wild giant pandas and four strains (Aimelvirus 2-5) from captive animals, with genome size of 8003-8100 bp. The six Aimelviruses shared 83-99.5\% sequence similarity (Fig. 4a) but showed no significant similarity with any viruses in GenBank over the full genomes based on BLASTn search. To investigate the relationship of the six Aimelviruses, a phylogenetic tree was established over the full-genome sequences (Fig. 4b), which showed that the six Aimelviruses were grouped into two separate clusters, one including three strains from captive giant pandas and the other one including one strain from captive giant pandas and two from the two wild giant pandas. In the lower cluster, although the two strains from the wild animals shared 99.5\% nucleotide sequence similarity, they only showed 91.4 and $90.2 \%$ similarities to the strain from the captive animals.

The genomic organization of the six Aimelviruses is typical of picornaviruses, with a single large ORF encoding the complete polyproteins of 2191-2218 amino acids (aa) in length. The polyprotein comprised the capsid proteins VP4, VP2, VP3, and VP1 and non-structural proteins $2 \mathrm{~A}$ to $2 \mathrm{C}$ and $3 \mathrm{~A}$ to $3 \mathrm{D}$ (Fig. $4 \mathrm{c}$ ). According to the ICTV (http://www.picornastudygroup.com/definitions/genus_definition.htm), the members of a picornavirus genus should share $>40,>40$, and $>50 \%$ amino acid similarity in their P1, P2, and P3 regions, respectively. The P1 regions of the six Aimelviruses shared 37.9$39.0 \%$ amino acid similarities with their closest relative, Saffold virus; the P2 and P3 regions of them shared $38.0-38.6 \%$ and $46.7-47.0 \%$ amino acid similarities, respectively, with their closest relative, the African bat icavirus. Therefore, according to the genetic distancebased criteria, the Aimelviruses from the giant pandas can be considered a novel genus in the family of Picornaviridae. Phylogenetic analyses were performed on the P1 and P3 regions of Aimelviruses here and 35 representative strains of all 35 genera in Picornaviridae, indicating that Aimelvirus was not significantly linked to any recognized or proposed genera (Fig. 3d and Additional file 3: Figure S1).

\section{A novel virus in Picornavirales order in feces of giant panda}

Members of the order Picornavirales are non-enveloped, single-stranded positive-sense RNA viruses found in a wide range of protozoa, plants, and animals. The order is currently composed of five recognized families: Picornaviridae, Secoviridae, Iflaviridae, Marnaviridae, and Dicistroviridae in addition to a large number of proposed, unassigned

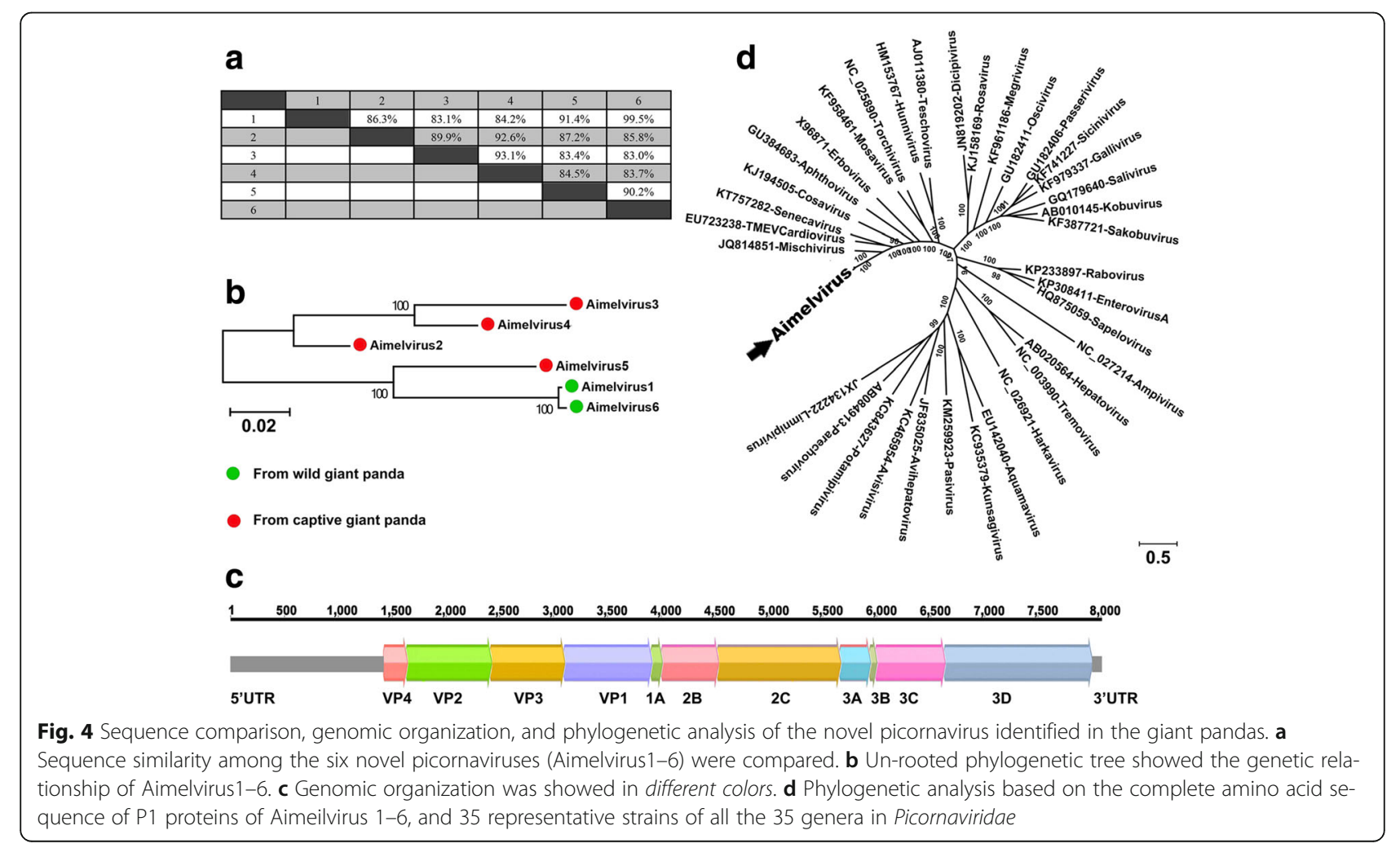


members. Conserved features found in all Picornavirales include non-structural proteins consisting of helicase, chymotrypsin-like protease, and RNA-dependent RNA polymerase (RdRp) domains. Genomic organization is variable with a single polyprotein expressed in all orders except Dicistroviridae where structural and non-structural protein genes are expressed from separate transcripts [29]. Recently, some highly divergent members of the Picornavirales order have been discovered, including porcine-stoolassociated RNA viruses (posaviruses) in the feces of pigs [30-32], fish-stool-associated RNA virus (fisavirus) in the intestinal content of a healthy carp [33], and human-stoolassociated virus (husavirus) [34].

In the present study, two large contigs, 8703 and 8612 bp, both encoding a 2778 aa polyprotein which showed sequence similarity to the polyprotein of posaviruses, were detected in the fecal samples from both wild giant pandas. We provisionally named the viruses Pansavirus 1 and Pansavirus 2 (panda-stool-associated RNA viruses 1 and 2). Based on the complete polyprotein, the two pansaviruses shared $82.1 \%$ amino acid identity to each other. In the polyprotein of Pansavirus 1, five conserved domains could be identified by an NCBI combined with a Pfam conserved domain search, while in the polyprotein of Pansavirus 2, only four conserved domains could be identified (Fig. 5a). The RdRp and capsid proteins of pansavirus both shared the highest amino acid identity of about $48 \%$ with the Posavirus strain 9676. The phylogenetic relationship was then determined by aligning the putative-encoded RDRP protein with representative members of the Picornavirales. Pansaviruses clustered together with other five posaviruses and a transcript from the nematode Ascaris suum, yet is clearly distant from posaviruses $1-3$, fisavirus, and husavirus (Fig. 5b).

\section{Anelloviruses in the blood samples of giant pandas}

Anelloviruses are non-enveloped, with circular singlestranded DNA genome of 2.1-3.9 kb in length depending
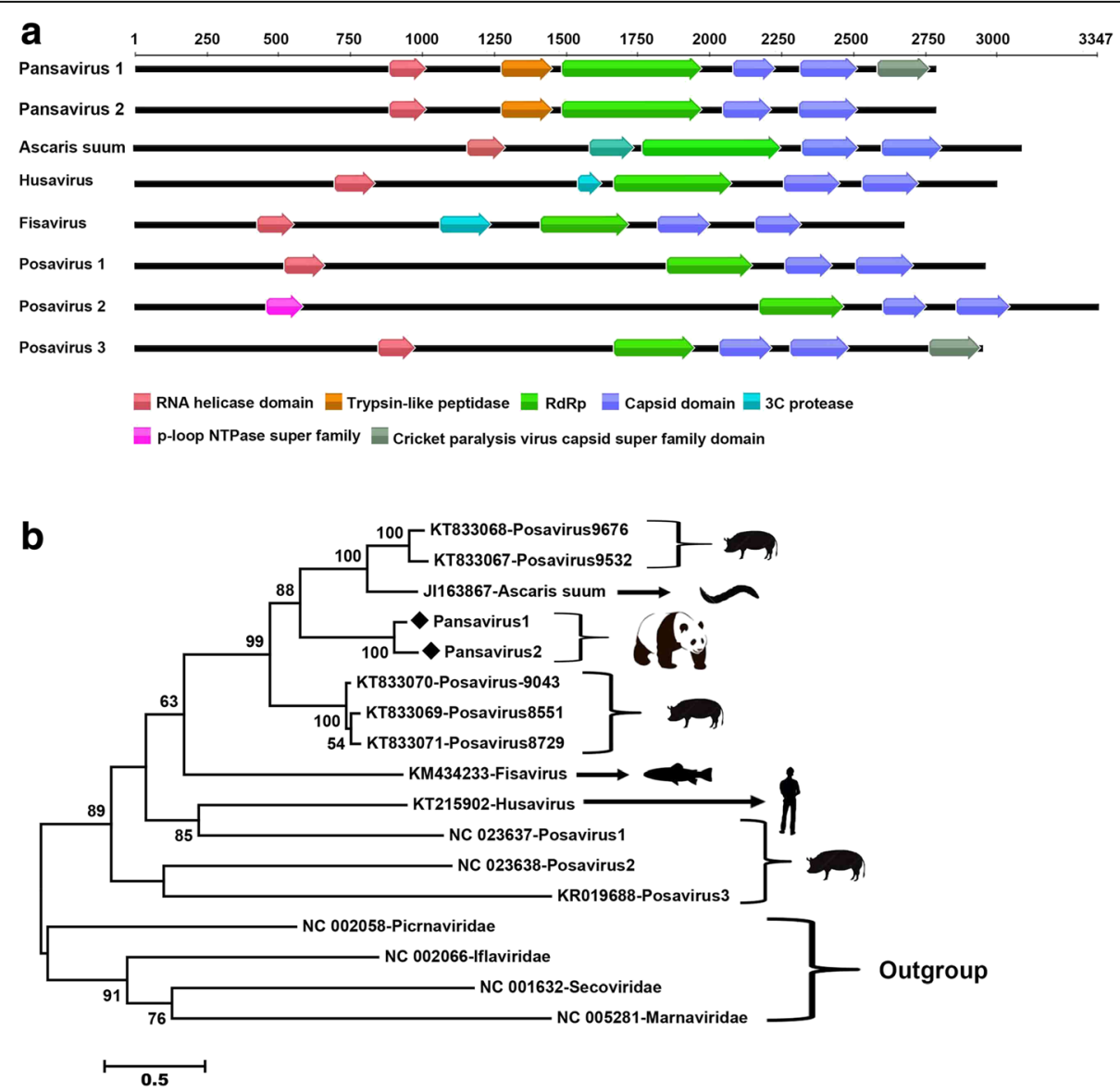

Fig. 5 Genomic organization and phylogenetic analysis of the novel picornavirales identified in the giant pandas. a Positions of conserved domains within the polyproteins of pansaviruses and their related viruses were shown in different colors. b Phylogenetic analysis was performed based on the amino acid sequence of RDRP protein. The sequence alignments included the two pansaviruses identified here and related strains in GenBank. Silhouettes of the hosts included in the phylogenetic analysis were showed on branches. Pansaviruses identified in this study were labeled with diamond 
on the isolate analyzed. Anelloviruses are subgrouped into 12 genera including 3 infecting humans, namely Alphatorquevirus or Torque teno virus (TTV), Betatorquevirus or Torque teno mini virus (TTMV), and Gammatorquevirus or Torque teno midi virus (TTMDV) [35]. Anelloviruses are widely prevalent in humans and animals and except for a possibly pathogenic porcine anellovirus [36] most appear to be commensal infections whose concentration increases with decreased immune function [37-39].

Here, abundant sequence reads were detected with translated amino acid similarity to viruses in the family Anelloviridae. We named the viruses Giant panda Anellovirus (GpAV). GpAV sequence reads were detected in all 12 blood samples, 4 of the 13 feces libraries, 2 of the 11 nasopharyngeal secretions, and 4 of the 5 tissues. From the 12 blood libraries abundant of anellovirus sequence reads, 19 complete genomes were got by de novo assembly combining inverse PCR and direct Sanger sequencing of the amplicons. The genome sizes of the GpAVs were ranged from 1964 to $2663 \mathrm{bp}$, whose circular genomes are depicted in Additional file 4: Figure S2. These genomes showed similar genomic organization in their ORF1, ORF2, and ORF3; although, ORF3 could not be detected in one genome and the locations of other theoretical ORFs of unknown function differed. ORF1 sequences of the 19 GpAVs ranged from 441 aa to 685 aa long, with typical arginine-rich regions at their N-termini. Sequence analysis indicated that the 19 GpAVs shared sequence identity of their ORF1 proteins ranging from 23 to $56 \%$.

Phylogenetic analyses were performed based on the ORF1 amino acid sequences of the 19 GpAVs, their best BLASTp matches in GenBank and the representative members of related viruses. Results indicated that the 19 GpAVs could be located in four different clades, including one, two, five, and $11 \mathrm{GpAV}$ strains, respectively (Fig. 6a). In order to detect coinfections in blood samples, the raw sequencing data of the 12 libraries from blood samples were aligned to each of the 19 complete genomes of GpAV, where the 19 genomes were used as reference genomes, the libraries with $>3$ different reads matching to the reference genome were considered as positive for the reference strain. High levels of co-infection of GpAV were detected in blood samples of giant pandas, with individual animals carrying highly distinct anellovirus variants (Fig. 6b). Co-infections were detected in 11 of the 12 blood samples. Library CB9 from a healthy captive animal had the highest level of co-infection, carrying 15 different GpAV strains. The library WB1 from the diseased wild giant panda was co-infected with 12 different GpAV strains (Fig. 6b).

\section{Circoviridae-like and Genomoviridae in multiple samples of Giant pandas}

Circoviridae, including recognized genera Circovirus and Cyclovirus are non-enveloped, single-stranded circular DNA $(\approx 2 \mathrm{~kb})$ viruses. Genomoviridae, currently consisting of a single genus Gemycircularvirus, is a new family of eukaryote-infecting single-stranded (ss) circular ssDNA viruses [40]. Circoviruses are thought to exhibit host species specificity and have been detected in various species, including birds and mammals, and associated with a variety of diseases particularly in pigs, including respiratory and enteric disease, dermatitis, and reproductive problems [41-44]. Gemycircularviruses were found in a wide range of organism and environments [44, 45]. Only one cellular host, a fungus [46], has been definitely identified for a single gemycircularvirus (SsHADV-1). Other gemycircularvirus genomes have been detected in fungi infected plants [47], sewage [48], the bodies of insects [45, 49], as well as mammalian feces and tissues [50, 51]. More recently, gemycircularviruses were described from human blood from blood donors [15], an HIV-positive patient [52], and human cerebral spinal fluid and feces [53]. Both circoviruses and gemycircularviruses have an ambisense genome organization with two major inversely arranged open reading frames encoding the rolling circle replication initiator protein gene (Rep) and a capsid protein gene (Cap). A conserved stem-loop structure, required for viral replication, is located between the $5^{\prime}$ ends of the two main ORFs.

Here, gemycircularvirus sequence reads were detected in feces, nasopharyngeal secretions and blood samples, while circovirus-related sequences were only detected in the fecal samples. Fifteen novel gemycircularvirus genomes, named giant panda gemycircularvirus (GpGmCV), and four genomes related to circoviruses, named giant panda circovirus-like viruses $(\mathrm{GpCV})$, were assembled from these libraries. Among the 15 genomes of gemycircularvirus, 14 were from fecal samples and one (GpGmCV14) from nasopharyngeal secretion. Among the $14 \mathrm{GpGmCV}$ genomes from fecal samples, 13 were from captive giant pandas and one (GpGmCV8) from the wild healthy giant panda (Fig. 7a). The four GpCV genomes were all from fecal samples, two of which were from the healthy wild giant panda while the other two from two different captive animals (Fig. 7a). The 15 genomes of GpGmCVs, with genome size of 2063 to 2240 bp long, have similar genome organizations, including a spliced Rep protein ORF, a Cap ORF in the opposite orientation, and a putative unknown protein on the same orientation of Rep (Fig. 7b). The stemloop structure was found between the $5^{\prime}$ ends of the two main ORFs of the genomes of all the 15 GpGmCVs, 12 of which contained a conserved nonamer 


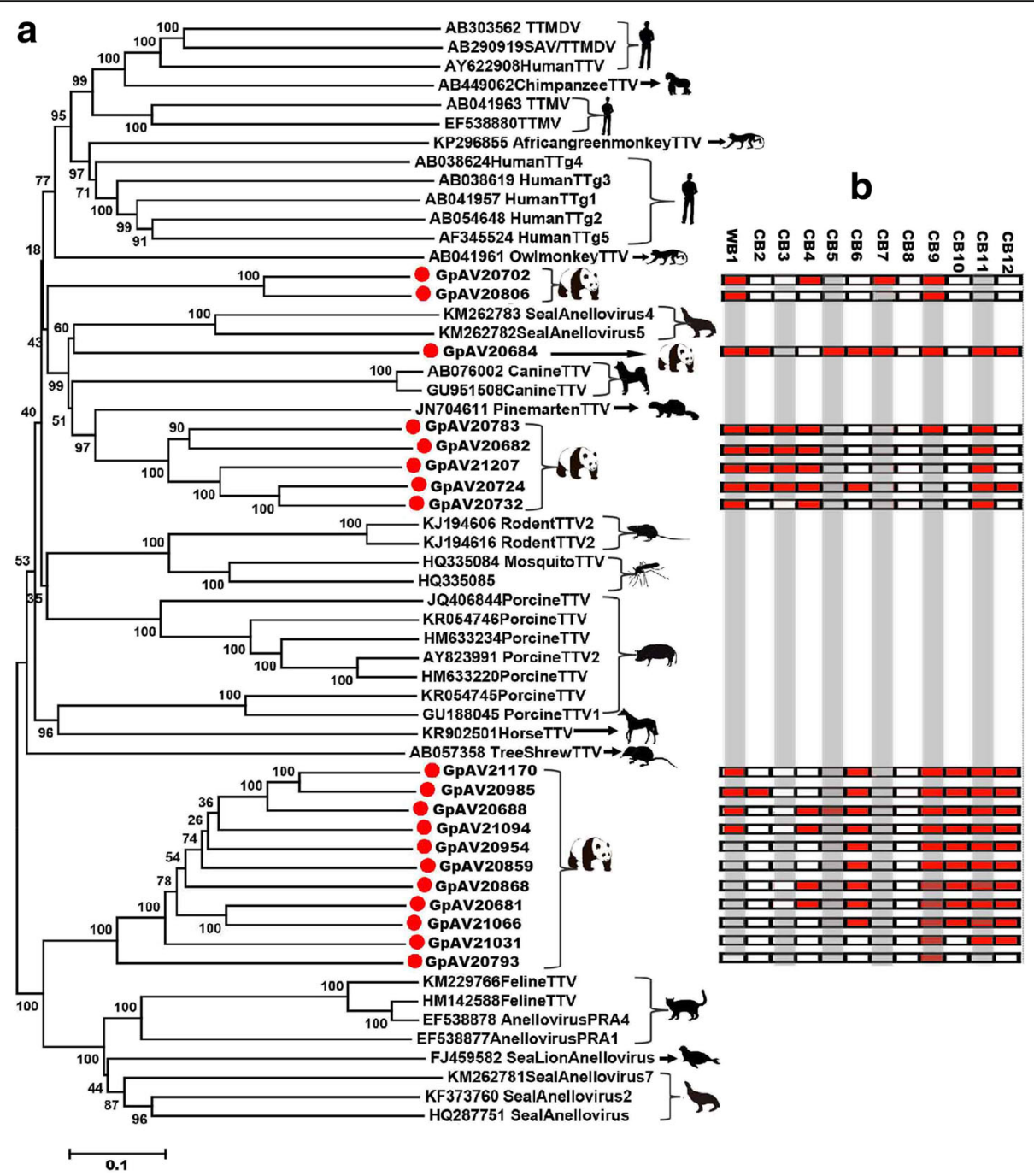

Fig. 6 Phylogenetic analysis and co-infection of the novel anelloviruses identified in the blood samples of giant pandas. a Phylogenetic analysis was performed based on the amino acid sequence of ORF1 protein. The sequence alignments included the 19 anelloviruses identified here, their best BLASTp matches in GenBank based on the ORF1 proteins, and the representative anellovirus strains from GenBank. Silhouettes of the hosts included in the phylogenetic analysis were showed on branches. Anelloviruses identified in this study were labeled with red dots. b The co-infection of anellovirus in blood giant pandas. The 12 columns and 19 rows were set corresponding to 12 blood samples and 19 anelloviruses, respectively, with complete genomes. The small box with red color stands for positive, and the white box stands for negative

of "TARTRTTK", while the other three have distinct base composition (Fig. 7c). The four genomes of GpCV have genome size of 1908 to $2745 \mathrm{bp}$ long, whose ORF organizations are different though they all contain the two mains proteins of Rep and Cap (Fig. 7d-g). GpCV1 and GpCV4 have the Rep protein and the Cap protein in the same orientation but GpCV2 and GpCV3 contain the two major proteins in the opposite orientation that is the classical genome feature of circovirus. The four GpCVs have different stemloop structures within the non-coding region between the ORFs of Rep and Cap, only two of which include the typical circovirus nonamer origin of replication (T/cAGTATTAC) (Fig. 7h).
In order to phylogenetically classify the GpGeCVs and GpCVs, their Rep proteins were aligned to those of circoviruses, cycloviruses, and gemycircularviruses. The phylogenetic analysis included the GpGeCVs and GpCVs identified in the present study and their best BLASTp matches based on Rep in GenBank, and representative strains of circoviruses, cycloviruses, and gemycircularviruses. Results indicated that the 15 GpGmCVs were located on 8 genetically different separate branches (Fig. 7a), sharing 45-87\% identities with their closest relatives. Although the four GpCVs were genetically very divergent based on the amino acid sequence of Rep, they nonetheless clustered together with four other putative circoviruses including 

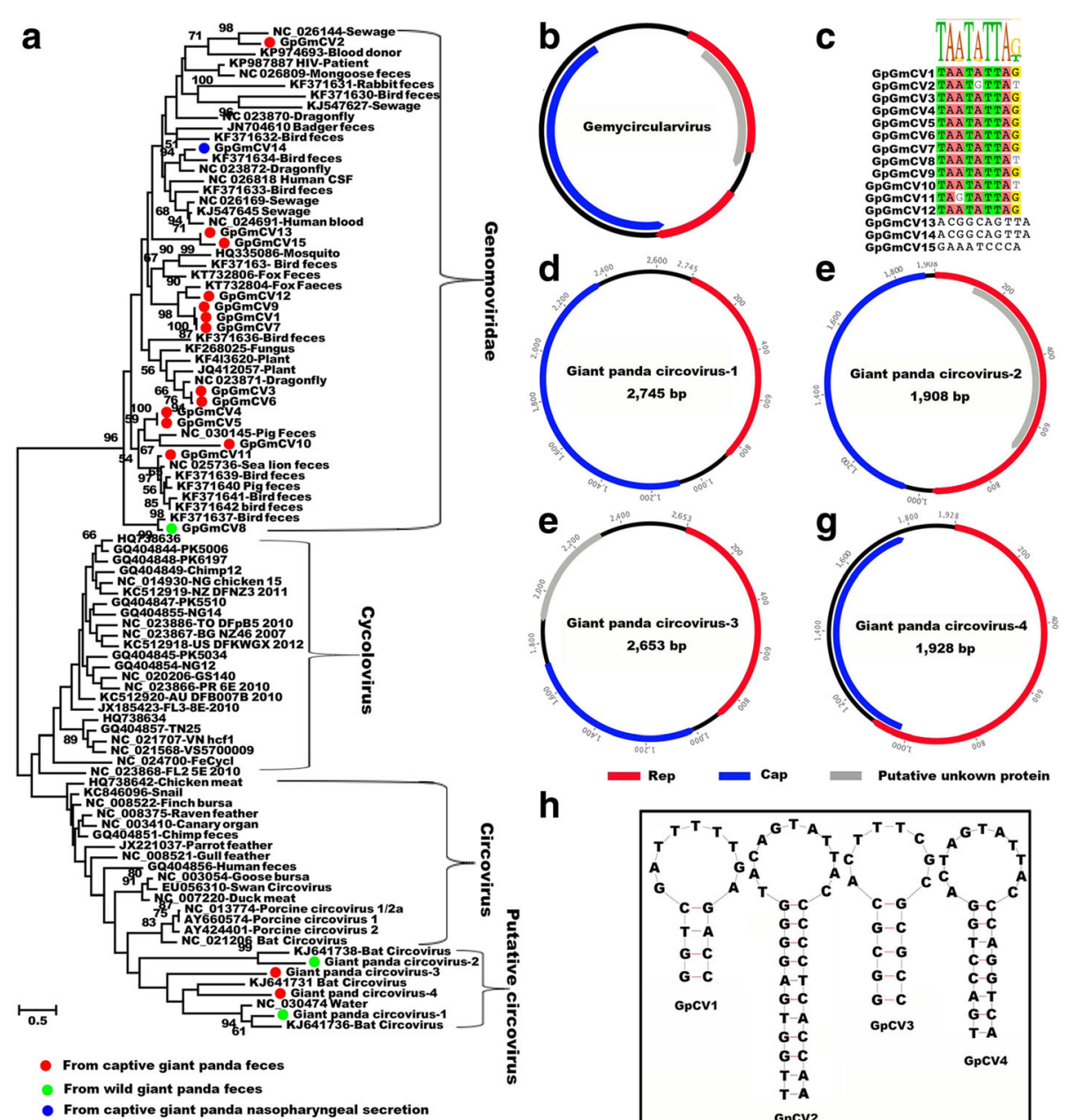

h

\begin{tabular}{|c|c|}
\hline 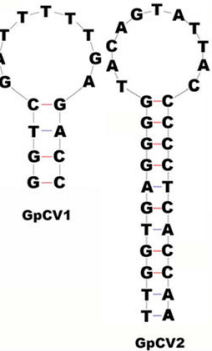 & 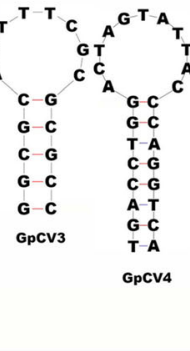 \\
\hline
\end{tabular}

Fig. 7 Phylogenetic analysis and genomic organization of the novel gemycircularviruses and putative circoviruses identified in the giant pandas. a Phylogenetic analysis was performed based on the amino acid sequence of Rep protein. The sequence alignments included 15 gemycircularviruses and 4 putative circoviruses identified here, their best BLASTp matches in GenBank based on the Rep proteins, and the representative strains of gemycircularvirus and circovirus. Hosts or sources of these viruses included in the phylogenetic analysis were showed on branches. Viruses identified in this study were labeled with colored dots. $\mathbf{b}$ The consensus genomic organization of the gemycircularviruses identified in giant pandas. $\mathbf{c}$ The nonamer in stem-loop structure of gemycircularviruses identified in this study. $\mathbf{d}-\mathbf{g}$ The genomic organizations of the four GpCVs. $\mathbf{h}$ The stem-loop structures of the four GpCVs identified in giant pandas

three circovirus-like viruses from bat and one circoviruslike virus from a Chinese lake sample, sharing 35-62\% amino acid sequence identity (Fig. 7a).

\section{RNA virus related to both picobirnavirus and partitivirus}

Picobirnavirus (PBV), the only genus in the new Picobirnaviridae viral family, has a bi-segmented dsRNA genome. The large RNA segment encodes the capsid protein while the small segment encodes the viral RdRp. PBV was originally found in the intestines of rat and has since been found in numerous mammals, birds, and reptiles [54]. Members of family Partitiviridae are characterized by having genomes comprised of two linear, monocistronic dsRNA segments (1.4 to $2.4 \mathrm{kbp}$ in length) [55], where the smaller codes for the $\mathrm{CP}$ and the larger codes for the virion-associated RNA polymerase. Most partitiviruses examined to date are associated with latent infections of their fungal, plant, and protozoan hosts [55].

In the present study, one fecal library (ID: 23FNC) contained 24 RdRp sequence reads that assembled into a full-length $\mathrm{RdRp}$ gene whose amino acid sequence showed approximately $30 \%$ identity to the viruses from both Paritiviridae and Picobirnaviridae. Alignment of full-length RdRp allowed the construction of a phylogenetic tree using four representative best BLASTx matches of partitivirus and picobirnavirus, in GenBank. This RdRp sequence phylogenetically falls between partitivirus and picobirnavirus (Fig. 8). Identification of a segment encoding a capsid gene failed, which may due to a 


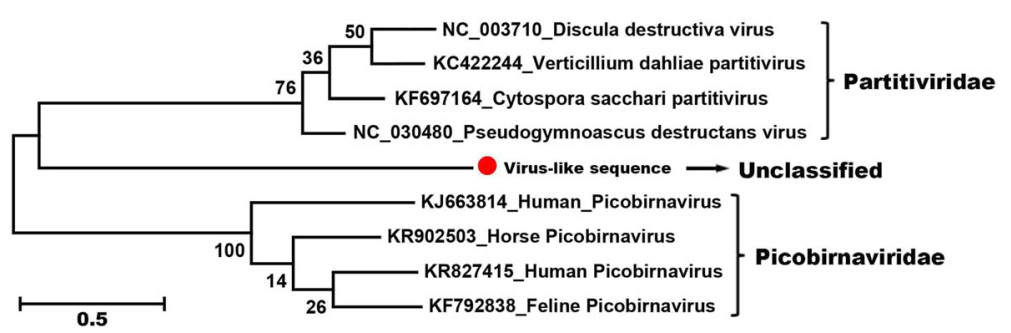

Fig. 8 Phylogenetic analysis of the partitivirus-like sequence identified in the giant pandas. Phylogenetic analysis was performed based on the amino acid sequence of RDRP protein. The sequence alignments included the partitivirus-like sequences identified in the giant pandas, four representative partitiviruses, and four representative picobirnaviruses. The partitivirus-like sequence identified in this study was labeled with a red dot

high level of sequence divergent. Sequence mapping using this $R d R p$ sequence as a reference to the raw data of this library showed that it is also present in other two fecal libraries. Whether this RdRp sequence belongs to the Partitiviridae or Picobirnaviridae families or is a new viral family is currently unknown.

\section{Other mammalian viruses}

Other viral genomes with high-sequence similarity to known viruses were also detected. Gyroviruses have been proposed as members of Anelloviridae as they share common genome characteristics [56]. Fifty-nine gyrovirus reads were detected in a fecal library (ID: FCN21) generated from three fecal samples from captive animals which could be assembled into three contigs. The complete genome was determined by inverse PCR, which contained $2375 \mathrm{bp}$ and shared the highest sequence identity of $97.8 \%$ with an avian gyrovirus (AGV2 GenBank: HM590588) detected in chicken serum [57] and human feces in China and $96 \%$ identity with a related genome found on human skin [58]. In the nasopharyngeal secretion sample from the sick wild giant panda, 15 sequence reads also showed similarity (70-90\%) to adenovirus proteins which could be assembled into five contigs ranged 272 to 472 bp matching five different gene regions of Mastadenovirus.

\section{Insect and plant viruses}

A large number of reads related to insect viruses were detected in all the fecal libraries. A smaller number of insect virus reads were detected in three nasopharyngeal secretion samples and the lung tissue (Table 2). Detection of these viral sequences in fecal samples may be due to insect consumption or the presence of intestinal parasites including nematodes. The presence of insect viral sequences in nasopharyngeal secretion samples and the lung tissue may be due to inhalation of insect-contaminated material or parasite infection in the respiratory tract. For instance, the largest contig ( $604 \mathrm{bp}$ ) assembled from the 29 reads in one nasopharyngeal secretion sample (ID: NSC9) showed amino acid sequence identity of $88 \%\left(E\right.$ value $\left.=4 \mathrm{e}^{-101}\right)$ with the structural protein of a Drosophila immigrans Nora virus (NC_024488). The insect genome in the lung tissue belongs to a divergent member of the Iflaviridae family, with the largest contig (600 bp) sharing an amino acid sequence identity of $30 \%\left(E\right.$ value $\left.=7 \mathrm{e}^{-21}\right)$ with the structural protein (VP1) of Sacbrood virus (KX254334). Overall, the insect RNA virus-like reads were more abundant than those of insect DNA viruses, making up $>70 \%$ of insect viral sequences. Most of the RNA sequences were related to the members of the Iflaviridae and Dicistroviridae. Insect DNA viruses were mainly from the viral families Iridoviridae, Baculoviridae, and Polydnaviridae and subfamily Densovirinae. The insect virus sequences shared amino acid sequence identity of $25-90 \%$ with annotated insect viral proteins.

Large numbers of plant virus sequences were detected in each fecal library but none in respiratory swabs, plasma, or tissues (Table 2). RNA viruses were predominant with $>80 \%$ of plant viral reads, the majority being related to single-stranded RNA viruses in the family Tombusviridae, followed by the families Partitiviridae, Secoviridae, Geminiviridae, Luteoviridae, Virgaviridae, and Rhabdoviridae, genus Umbravirus, and family Alphaflexiviridae. Most of the plant DNA viral reads were related to members of the Phycodnaviridae family.

\section{Discussion}

Animal and human viral discovery has long been focused on pathogenic infections and viruses that could be readily grown in cell cultures and cause visible cytopathic effects. Viral metagenomics is a recent approach to analyzing mixtures of viral nucleic acids enriched directly from a variety of sources without a prerequisite for amplification in tissue culture. Viral metagenomics has recently been used in numerous animal virus discoveries [14, 30, 59-62], providing information on the composition of animal viromes, helping to provide candidates to identify the etiology 
of infectious disease in animals and identify zoonotic and emerging viruses. We describe here the eukaryotic viral communities in the feces, blood, nasopharyngeal secretion, and five tissues of giant pandas. The animals analyzed here consisted of an extremely emaciated wild giant panda who died of an unknown cause, one healthy wild giant panda, and 46 healthy captive giant pandas. The highest percentage reads of a new picornavirus in feces and of anelloviruses in plasma (19 different variants) were detected in the sick animal relative to all healthy giant pandas. The nasopharyngeal swab of the diseased animal also generated the largest percentage of papillomavirus reads relative to the only two papillomavirus positive healthy animals. A low level of adenovirus reads was also found in nasopharyngeal secretion. The nasopharyngeal swab of the diseased animal contained three mammalian viruses (two types of papillomavirus, anellovirus, and adenovirus), higher than those of the healthy captive giant pandas with an average of 0.9 distinct mammalian viruses per animal (with co-infection ranging from 0 to 3 ). The diseased animal was co-infected with at least 12 different anelloviruses, a greater number than the average of seven distinct anellovirus per animal (range 0-15).

The picornavirus and papillomavirus stood out as two viruses whose sequence reads percentages were much higher than those of the healthy giant pandas. Increases in viral sequence reads concentration might reflect weakened immune status of the diseased animal as is known to occur with anelloviruses in humans [37-39]. Although serologic test indicated that this sick wild giant panda was weakly positive for canine parvovirus antibodies, none of its samples showed the presence of canine parvovirus sequences.

Papillomaviruses are believed that have co-evolved with their vertebrate host species, a hypothesis supported by the fact that PVs of closely related host species are generally closely related themselves [63, 64]. In the present study, four PVs (AmPV1-4) belonging to three different genera were detected in the nasopharyngeal secretion of the sick wild and two captive giant pandas. AmPV1 and AmPV2 were detected in the nasopharyngeal secretion sample of the diseased giant panda and phylogenetically clustered with the UmPV both in the Omegapapillomavirus genus (Fig. 3b) from the oral mucosa of a polar bear with papillomas on the tongue [27]. The two different AmPVs generated sequences accounting for a large 7.07\% of the unique sequence reads (Fig. 2c), which may have contributed to the mouth mucous membrane canker of the dying wild giant panda [65]. A Lambdapapillomavirus was recently associated with oral papillomas in southern sea otters which may have interfered with their feeding capacity [66]. AmPV3 and AmPV4 were found in two nasopharyngeal secretion samples from two captive giant pandas, respectively. AmPV4 showed the highest sequence similarity to PIPV detected in the a cutaneous lesion biopsy of a raccoon [67], both of which phylogenetically grouped into the genus Lambdapapillomavirus also containing CPV1 from dogs and FcaPV1 from cats (Fig. 3b). AmPV3, although clustering with PVs from rat and sea lions (Fig. 3b), may represent a new genus in Papillomaviridae family based on ICTV genetic distance criteria. The AmPV1, AmPV2, and AmPV4 clustered with their closest relatives, UmPV and PIPV, whose hosts, polar bears and raccoons are close relative of giant panda. With the increasing number of PVs discovery from animals, we anticipate that the relative of AmPV3 will be detected in hosts closely related to giant pandas.

The genome of a tentative new picornaviridae genus, provisionally labeled Aimelvirus, was characterized. The greatest sequence similarity values were seen with members of the genera Mischivirus, Cardiovirus, and Senecavirus. We generated six complete or near complete genomes of Aimelviruses. These genomes could be classified into two potential species, each with three strains. Two strains from two wild animals clustered together showing high sequence identity (99.5\%) and $91.4 \%$ to the other strain in the same clade from a captive animal (Fig. 4a). The three strains in the other group were all from the captive giant pandas and showed more sequence divergence, sharing sequence similarity of $89.9-93.1 \%$ among themselves. All fecal libraries, including eight libraries constructed from small sample pools and five from individual sample, were positive for Aimelvirus (Table 2), suggesting that giant pandas are frequent natural hosts of this picornavirus. None of the blood samples were positive for Aimelvirus.

The order Picornavirales includes viruses replicating in all the major divisions of eukaryotes, including protozoans, plants, insects, and mammals [68]. In the current study, two novel viral RNA genomes with distant sequence relationships to Picornavirales were characterized and provisionally named pansaviruses. Phylogenetic analysis based on the amino acid sequence of RdRp indicated that these genomes clustered together with those of posaviruses from pig feces and the transcriptome of an Ascaris suum transcriptome (Fig. 5b) [32]. Since an Ascaris suum transcriptome sequence was also grouped into this cluster, pansaviruses may not actually infect the intestinal cells of the giant panda but a nematode species in the gut of these animals, a potential source supported by the clinical record that the diseased giant panda contained ascarids in its intestine. The possible origin of posaviruses from pigs from aquatic algae has also been postulated based on their similarity to members of the algae infecting Marnaviridae and detection in water from wells on pig farms [32]. Only one fecal sample from the 25 captive giant pandas was positive for pansavirus, 
possibly reflecting the different environment in a breeding center relative to the wild.

In recent years, many genomes of rep-containing circular DNA viruses in the Circoviridae and Genomoviridae have been characterized in mammals, birds, insects, fungi, and environmental samples bringing to light a high level of genetic diversity among these viruses $[40,44,56]$. In this work, we reported the discovery of 19 novel circular ssDNA genomic sequences from giant panda, 15 belonging to gemycircularvirus in Genomoviridae and 4 possibly belonging to circovirus in Circoviridae. Gemycircularviruses have been reported in feces of a wide range of organism and different environments [40, 45]; however, the host species of these viruses are not known except for one replicating in a fungi. In this study, based on the Rep protein sequence, some gemycircularviruses from giant panda samples showed high sequence identity to the viruses detected in insects or feces from other animals (Fig. 7a), e.g., GpGmCV3 and GpGmCV6 sharing identity of $81 \%$ with the gemycircularvirus from dragonfly (NC_023871) [45] and GpGmCV8 sharing $87 \%$ sequence identity with gemycircularvirus from bird feces (KF371637) [69], which suggested that the detection of gemycircularvirus genomes in giant panda may be from consumed fungi or insects. As for the four putative circoviruses, two (GpCV3 and GpCV4) were from captive giant pandas and the other two (GpCV1 and GpCV2), from the healthy wild giant panda.

Anelloviruses from humans and animals show a very high level of genetic diversity [70-72]. Co-infections with multiple anellovirus variants simultaneously present in the same individual and animals have been described [73-76]. As induced, infectious, or inborn immunodeficiency is associated with increases in viral loads, anelloviruses are believed to be under immunological control [37, 38, 77, 78]. Anelloviruses have been reported in many tissues and bodily fluids, including respiratory fluids, blood, breast milk, cervical secretions, semen, urine, and feces [35]. In the present study, Anellovirus were detected in all studied sample types, with blood samples showing the highest sequence reads percentage. All the 12 blood samples were positive for anellovirus, 11 of which revealed co-infections. The diseased wild animal showed the highest percentage anellovirus sequence reads (11.8\%) which may reflect this giant panda's poor immunity in the late stage of its disease.

The virome is one part of the microbiome in mammals which is being continuously updated using viral metagenomic sequencing. The present study analyzed the viromes of a wild-diseased and multiple healthy giant pandas. The same viral families were detected in both the dying and the healthy animals; although, the fraction of papillomavirus, anellovirus, and picornavirus were greater in the affected animal. It is increasingly clear that the presence of viruses is not necessarily indicative of pathogenesis and instead may represent a degree of commensal interactions between virus and host [79]. It is difficult to know how stably associated the virome is with the host, as some viruses rely solely on a lytic life cycle and tend to produce short-lived acute infections. However, non-cytopathic viruses, which do not cause such profound cell death, can infect and persist for the lifespan of the host. In the present study, the Aimelvirus and Anellovirus were detected in all of the studied giant pandas including the healthy and sick animals. The replication of these viruses may increase if the host has low immunity [38], and further worsen the host's condition. In this study, the virus titers of anelloviruses in the blood and Aimelvirus in the gut of the sick giant panda were distinctly higher than those from the normal giant pandas (Fig. 3), suggesting that the balance between the immune control and viral replication was compromised, leading to the higher viral loads in the already sick giant panda.

\section{Conclusion}

Our study provides an overview of the virome of the giant pandas and significantly increases the diversity of viruses known to infect this vulnerable species. Comparison of the viruses in the healthy animals with those in future giant panda suffering sporadic or epidemic unexplained diseases of possible viral origins will help identify newly emerging, possibly pathogenic, viruses. Higher read number of papillomaviruses, anelloviruses, and picornaviruses in the emaciated, sick, animal may reflect reduced immune control of otherwise commensal infections.

\section{Additional files}

Additional file 1: Table S1. Primers used for specific PCR confirmation and inverse PCR. (DOCX $24 \mathrm{~kb}$ )

Additional file 2: Table S2. Genome size, GC content, and classic elements of papillomavirus identified in giant pandas. Classic elements included positions of Zinc-binding domains of E6 and E7 ORFs, LXCXE motif of E7 ORF, ATP binding site of the ATP-dependent helicase (GPPDTGKS), E2-binding site motifs, TATA box, and polyadenylation site of URR. (DOCX $18 \mathrm{~kb}$ )

Additional file 3: Figure S1. Phylogenetic tree based on the P3 protein of Aimelvirus. Phylogenetic analysis was performed based on the complete amino acid sequence of P3 proteins of Aimeilvirus 1-6 and 35 representative strains of all the 35 genera in Picornaviridae. The Aimelvirus identified in this study was labeled with a black dot. (JPEG 368 kb)

Additional file 4: Figure S2. Genome structure of anelloviruses identified in the present study. (JPEG $1012 \mathrm{~kb}$ ) 


\section{Abbreviations}

AmPV: Ailuropoda melanoleuca papillomavirus; BLAST: Basic Local Alignment Search Tool; GpAV: Giant panda anellovirus; GpCV: Giant panda circovirus; GpGmCV: Giant panda gemycircularvirus

\section{Acknowledgements}

We would like to thank Mr. Limin Chen and Mr. Weichao Zheng in the Tangjiahe Nature Reserve, Mr. Zhi Yang in the Chengdu Research Base of Giant Panda Breeding, and Mr. Xiaodong Gu and Mr. Xuyu Yang in the Forestry Department of Sichuan Province for their help in collecting samples.

\section{Funding}

This work was partly supported by the National Key Research and Development Programs of China No. 2017 YFC1200201 and No. 2016YFC0503200; National Natural Science Foundation of China Nos. 31302107, 31372223, 31572525 and 31402211; Jiangsu Provincial Key Research and Development Projects No.BE2017693; Natural Science Foundation of Jiangsu Province Nos. BK20140537 and BK20140578; Sichuan Youth Science and Technology Foundation No. 2017JQ0026; Chengdu Giant Panda Breeding Research Foundation Nos. CPF 2013-17, 2014-11, and 201519; Agri-X foundation of Shanghai Jiaotong University in 2014; and Blood Systems Research Institute and National Institutes of Health no. R01 Al123376.

\section{Availability of data and materials}

The raw sequence reads from the metagenomic libraries were deposited in the Short Read Archive of GenBank database under accession number: SRX2882233.

\section{Authors' contributions}

$W Z, D Q$, and $\mathrm{RH}$ conceived the idea. All authors contributed to study design. WZ, SY, ZL, XW, XT, GL, and ED conducted the study and analyzed the data. WZ, DQ and ED wrote the manuscript, and all authors edited the manuscript. All authors read and approved the final manuscript.

\section{Ethics approval and consent to participate}

The sample collection and all the experiments in the present study were performed with an ethical approval given by Ethics Committee of Jiangsu University and the reference number is No. UJS2014017.

\section{Consent for publication}

Not applicable.

\section{Competing interests}

The authors declare that they have no competing interests.

\section{Publisher's Note}

Springer Nature remains neutral with regard to jurisdictional claims in published maps and institutional affiliations.

\section{Author details}

'Department of Microbiology, School of Medicine, Jiangsu University, Zhenjiang, Jiangsu 212013, China. ${ }^{2}$ Sichuan Key Laboratory of Conservation Biology for Endangered Wildlife, Chengdu Research Base of Giant Panda Breeding, Chengdu, Sichuan 610081, China. ${ }^{3}$ Department of Swine Infectious Disease, Shanghai Veterinary Research Institute, Chinese Academy of Agricultural Sciences, Shanghai 200241, China. ${ }^{4}$ Department of Laboratory Medicine, Jiangsu Taizhou People's Hospital, Taizhou, Jiangsu 225300, China. ${ }^{5}$ School of Agriculture and Biology, Shanghai Jiaotong University, Shanghai 200240, China. ${ }^{6}$ Blood Systems Research Institute, Department of Laboratory Medicine, University of California San Francisco, San Francisco, CA 94118, USA. ${ }^{7}$ Sichuan Key Laboratory of Conservation Biology for Endangered Wildlife, Chengdu Research Base of Giant Panda Breeding, Chengdu, Sichuan 610000, China
Received: 6 October 2016 Accepted: 13 July 2017

Published online: 07 August 2017

\section{References}

1. Wei F, Hu Y, Zhu L, Bruford MW, Zhan X, Zhang L. Black and white and read all over: the past, present and future of giant panda genetics. Mol Ecol. 2012:21:5660-74.

2. Krause J, Unger T, Noçon A, Malaspinas A-S, Kolokotronis S-O, Stiller M, Soibelzon L, Spriggs H, Dear PH, Briggs AW, Bray SCE, O'Brien SJ, Rabeder G, Matheus P, Cooper A, Slatkin M, Pääbo S, Hofreiter M. Mitochondrial genomes reveal an explosive radiation of extinct and extant bears near the Miocene-Pliocene boundary. BMC Evol Biol. 2008;8:220.

3. Zhu L, Hu Y, Qi D, Wu H, Zhan X, Zhang Z, Bruford MW, Wang J, Yang X, Gu X, Zhang L, Zhang B, Zhang S, Wei F. Genetic consequences of historical anthropogenic and ecological events on giant pandas. Ecology. 2013;94:2346-57.

4. Xue Z, Zhang W, Wang L, Hou R, Zhang M, Fei L, Zhang X, Huang H, Bridgewater LC, Jiang $Y$, Jiang $C$, Zhao L, Pang $X$, Zhang $Z$. The bamboo-eating giant panda harbors a carnivore-like gut microbiota, with excessive seasonal variations. MBio. 2015:6:e00022-15.

5. Wei G, Lu H, Zhou Z, Xie H, Wang A, Nelson K, Zhao L. The microbial community in the feces of the giant panda (Ailuropoda melanoleuca) as determined by PCR-TGGE profiling and clone library analysis. Microb Ecol. 2007:54:194-202.

6. Zhu L, Wu Q, Dai J, Zhang S, Wei F. Evidence of cellulose metabolism by the giant panda gut microbiome. Proc Natl Acad Sci U S A. 2011;108:17714-9.

7. Williams CL, Dill-McFarland KA, Vandewege MW, Sparks DL, Willard ST, Kouba AJ, Suen G, Brown AE. Dietary shifts may trigger dysbiosis and mucous stools in giant pandas (Ailuropoda melanoleuca). Front Microbiol. 2016;7:661.

8. Wei F, Wang $X$, Wu Q. The giant panda gut microbiome. Trends Microbiol. 2015;23:450-2.

9. $\quad$ Feng $N$, Yu Y, Wang T, Wilker $P$, Wang J, Li Y, Sun Z, Gao Y, Xia X. Fatal canine distemper virus infection of giant pandas in China. Sci Rep. 2016;6:27518

10. Guo L, Yang S, Chen S, Zhang Z, Wang C, Hou R, Ren Y, Wen X, Cao S, Guo W, Hao Z, Quan Z, Zhang M, Yan Q. Identification of canine parvovirus with the Q370R point mutation in the VP2 gene from a giant panda (Ailuropoda melanoleuca). Virol J. 2013;10:163

11. Li D, Zhu L, Cui H, Ling S, Fan S, Yu Z, Zhou Y, Wang T, Qian J, Xia X, Xu Z, Gao Y, Wang C. Influenza A(H1N1)pdm09 virus infection in giant pandas, China. Emerg Infect Dis. 2014;20:480-3.

12. Edwards RA, Rohwer F. Viral metagenomics. Nat Rev Microbiol. 2005;3:504-10.

13. Delwart E. Animal virus discovery: improving animal health, understanding zoonoses, and opportunities for vaccine development. Curr Opin Virol. 2012;2:344-52.

14. Zhang W, Li L, Deng X, Kapusinszky B, Pesavento PA, Delwart E. Faecal virome of cats in an animal shelter. J Gen Virol. 2014;95(Pt 11):2553-64.

15. Zhang W, Li L, Deng X, Blümel J, Nübling CM, Hunfeld A, Baylis SA, Delwart E. Viral nucleic acids in human plasma pools. Transfusion. 2016;56:2248-55.

16. Liu Z, Yang S, Wang Y, Shen Q, Yang Y, Deng X, Zhang W, Delwart E. Identification of a novel human papillomavirus by metagenomic analysis of vaginal swab samples from pregnant women. Virol J. 2016;13:122

17. Deng X, Naccache SN, Ng T, Federman S, Li L, Chiu CY, Delwart EL. An ensemble strategy that significantly improves de novo assembly of microbial genomes from metagenomic next-generation sequencing data. Nucleic Acids Res. 2015:43(7):e46.

18. Skewes-Cox P, Sharpton TJ, Pollard KS, DeRisi UL. Profile hidden Markov models for the detection of viruses within metagenomic sequence data. PLoS One. 2014;9:e105067.

19. Johnson LS, Eddy SR, Portugaly E. Hidden Markov model speed heuristic and iterative HMM search procedure. BMC Bioinformatics. 2010;11:431.

20. Eddy SR. A new generation of homology search tools based on probabilistic inference. Genome inform. 2009:23:205-11.

21. Finn RD, Clements J, Eddy SR. HMMER web server: interactive sequence similarity searching. Nucleic Acids Res. 2011:39(Web Server issue):W29-37.

22. Finn RD, Bateman A, Clements J, Coggill P, Eberhardt RY, Eddy SR, Heger A, Hetherington K, Holm L, Mistry J, Sonnhammer ELL, Tate J, Punta M. Pfam: the protein families database. Nucleic Acids Res. 2014;42:D222-30.

23. Marchler-Bauer A, Derbyshire MK, Gonzales NR, Lu S, Chitsaz F, Geer LY, Geer RC, He J, Gwadz M, Hurwitz DI, Lanczycki CJ, Lu F, Marchler GH, Song JS, Thanki N, 
Wang Z, Yamashita RA, Zhang D, Zheng C, Bryant SH. CDD: NCBl's conserved domain database. Nucleic Acids Res. 2015;43(Database issue):D222-6.

24. Bernard H-U, Burk RD, Chen Z, van Doorslaer K, zur Hausen $H$, de Villiers E-M. Classification of papillomaviruses (PVs) based on 189 PV types and proposal of taxonomic amendments. Virology. 2010;401:70-9.

25. Joh J, Jenson AB, King W, Proctor M, Ingle A, Sundberg JP, Ghim S. Genomic analysis of the first laboratory-mouse papillomavirus. I Gen Virol. 2011;92(Pt 3):692-8.

26. Munday JS. Bovine and human papillomaviruses: a comparative review. Vet Pathol. 2014;51:1063-75.

27. Stevens H, Rector A, Bertelsen MF, Leifsson PS, Van Ranst M. Novel papillomavirus isolated from the oral mucosa of a polar bear does not cluster with other papillomaviruses of carnivores. Vet Microbiol. 2008;129:108-16.

28. Munday JS, Thomson N, Dunowska M, Knight CG, Laurie RE HS. Genomic characterisation of the feline sarcoid-associated papillomavirus and proposed classification as Bos taurus papillomavirus type 14. PubMed-NCBI Vet Microbiol. 2015:177:289-95.

29. Le Gall O, Christian P, Fauquet CM, King AMQ, Knowles NJ, Nakashima N, Stanway G, Gorbalenya AE. Picornavirales, a proposed order of positivesense single-stranded RNA viruses with a pseudo- $\mathrm{T}=3$ virion architecture. Arch Virol. 2008;153:715-27.

30. Shan T, Li L, Simmonds P, Wang C, Moeser A, Delwart E. The fecal virome of pigs on a high-density farm. J Virol. 2011;85:11697-708.

31. Hause BM, Hesse RA, Anderson GA. Identification of a novel Picornavirales virus distantly related to posavirus in swine feces. Virus Genes. 2015;51:144-7.

32. Hause BM, Palinski R, Hesse R, Anderson G. Highly diverse posaviruses in swine faeces are aquatic in origin. J Gen Virol. 2016;97:1362-7.

33. Reuter G, Pankovics P, Delwart E, Boros Á. A novel posavirus-related singlestranded RNA virus from fish (Cyprinus carpio). Arch Virol. 2015;160:565-8.

34. Oude Munnink BB, Cotten M, Deijs M, Jebbink MF, Bakker M, Farsani SMJ, Canuti M, Kellam P, van der Hoek L. A novel genus in the order Picornavirales detected in human stool. J Gen Virol. 2015:96:3440-3.

35. Spandole S, Cimponeriu D, Berca LM, Mihăescu G. Human anelloviruses: an update of molecular, epidemiological and clinical aspects. Arch Virol. 2015;160:893-908.

36. Aramouni M, Kekarainen T, Ganges L, Tarradas J, Segalés J. Increased viral load and prevalence of Torque teno sus virus 2 (TTSuV2) in pigs experimentally infected with classical swine fever virus (CSFV). Virus Res. 2013;172:81-4.

37. De Vlaminck I, Khush KK, Strehl C, Kohli B, Luikart H, Neff NF, Okamoto J, Snyder TM, Cornfield DN, Nicolls MR, Weill D, Bernstein D, Valantine HA, Quake SR. Temporal response of the human virome to immunosuppression and antiviral therapy. Cell. 2013;155:1178-87.

38. Li L, Deng $X$, Linsuwanon $P$, Bangsberg D, Bwana MB, Hunt P, Martin JN Deeks SG, Delwart E. AIDS alters the commensal plasma virome. J Virol. 2013;87:10912-5

39. Young JC, Chehoud C, Bittinger K, Bailey A, Diamond JM, Cantu E, Haas AR, Abbas A, Frye L, Christie JD, Bushman FD, Collman RG. Viral metagenomics reveal blooms of anelloviruses in the respiratory tract of lung transplant recipients. Am J Transplant. 2015;15:200-9.

40. Krupovic M, Ghabrial SA, Jiang D, Varsani A. Genomoviridae: a new family of widespread single-stranded DNA viruses. Arch Virol. 2016;161:2633-43.

41. Li L, Kapoor A, Slikas B, Bamidele OS, Wang C, Shaukat S, Masroor MA, Wilson ML, Ndjango J-BN, Peeters M, Gross-Camp ND, Muller MN, Hahn BH, Wolfe ND, Triki H, Bartkus J, Zaidi SZ, Delwart E. Multiple diverse circoviruses infect farm animals and are commonly found in human and chimpanzee feces. J Virol. 2010;84:1674-82.

42. Li L, McGraw S, Zhu K, Leutenegger CM, Marks SL, Kubiski S, Gaffney P, Dela Cruz FN, Wang C, Delwart E, Pesavento PA. Circovirus in tissues of dogs with vasculitis and hemorrhage. Emerg Infect Dis. 2013;19:534-41.

43. Opriessnig T, Halbur PG. Concurrent infections are important for expression of porcine circovirus associated disease. Virus Res. 2012;164:20-32

44. Delwart E, Li L. Rapidly expanding genetic diversity and host range of the Circoviridae viral family and other Rep encoding small circular ssDNA genomes. Virus Res. 2012;164:114-21.

45. Rosario K, Dayaram A, Marinov M, Ware J, Kraberger S, Stainton D, Breitbart M, Varsani A. Diverse circular ssDNA viruses discovered in dragonflies (Odonata: Epiprocta). J Gen Virol. 2012;93(Pt 12):2668-81.

46. Yu X, Li B, Fu Y, Jiang D, Ghabrial SA, Li G, Peng Y, Xie J, Cheng J, Huang J, Yi X. A geminivirus-related DNA mycovirus that confers hypovirulence to a plant pathogenic fungus. Proc Natl Acad Sci U S A. 2010;107:8387-92.
47. Rombaldi RL, Serafini EP, Mandelli J, Zimmermann E, Losquiavo KP. Transplacental transmission of human Papillomavirus. Virol J. 2008;5:106.

48. Kraberger S, Argüello-Astorga GR, Greenfield LG, Galilee C, Law D, Martin DP, Varsani A. Characterisation of a diverse range of circular replication-associated protein encoding DNA viruses recovered from a sewage treatment oxidation pond. Infect Genet Evol. 2015;31:73-86.

49. Dayaram A, Potter KA, Pailes R, Marinov M, Rosenstein DD, Varsani A. Identification of diverse circular single-stranded DNA viruses in adult dragonflies and damselflies (Insecta: Odonata) of Arizona and Oklahoma, USA. Infect Genet Evol. 2015;30:278-87.

50. van den Brand JMA, van Leeuwen M, Schapendonk CM, Simon JH, Haagmans BL, Osterhaus ADME, Smits SL. Metagenomic analysis of the viral flora of pine marten and European badger feces. J Virol. 2012;86:2360-5.

51. Lamberto I, Gunst K, Müller H, Zur Hausen H, de Villiers E-M. Mycovirus-like DNA virus sequences from cattle serum and human brain and serum samples from multiple sclerosis patients. Genome Announc. 2014;2(4): e00848-14. doi: 10.1128/genomeA.00848-14.

52. Uch R, Fournier P-E, Robert C, Blanc-Tailleur C, Galicher V, Barre R, Jordier F, de Micco P, Raoult D, Biagini P. Divergent Gemycircularvirus in HIV-positive blood, France. Emerg Infect Dis. 2015;21:2096-8.

53. Phan TG, Mori D, Deng X, Rajindrajith S, Ranawaka U, Fan Ng TF, BucardoRivera F, Orlandi P, Ahmed K, Delwart E. Small circular single stranded DNA viral genomes in unexplained cases of human encephalitis, diarrhea, and in untreated sewage. Virology. 2015;482:98-104.

54. Ganesh B, Masachessi G, Mladenova Z. Animal picobirnavirus. Virusdisease. 2014;25:223-38

55. Nibert ML, Ghabrial SA, Maiss E, Lesker T, Vainio EJ, Jiang D, Suzuki N. Taxonomic reorganization of family Partitiviridae and other recent progress in partitivirus research. Virus Res. 2014;188:128-41.

56. Rosario K, Duffy S, Breitbart M. A field guide to eukaryotic circular singlestranded DNA viruses: insights gained from metagenomics. Arch Virol. 2012;157:1851-71.

57. Rijsewijk FAM, Dos Santos HF, Teixeira TF, Cibulski SP, Varela APM, Dezen D, Franco AC, Roehe PM. Discovery of a genome of a distant relative of chicken anemia virus reveals a new member of the genus Gyrovirus. Arch Virol. 2011;156:1097-100.

58. Sauvage V, Cheval J, Foulongne V, Gouilh MA, Pariente K, Manuguerra JC, Richardson J, Dereure O, Lecuit M, Burguiere A, Caro V, Eloit M. Identification of the first human gyrovirus, a virus related to chicken anemia virus. J Virol. 2011;85:7948-50.

59. Li L, Giannitti F, Low J, Keyes C, Ullmann LS, Deng X, Aleman M, Pesavento PA, Pusterla N, Delwart E. Exploring the virome of diseased horses. J Gen Virol. 2015;96:2721-33.

60. Scheel TK, Simmonds PKA. Surveying the global virome: identification and characterization of HCV-related animal hepaciviruses. Antivir Res. 2015;115:83-93.

61. Wu Z, Yang L, Ren X, He G, Zhang J, Yang J, Qian Z, Dong J, Sun L, Zhu Y, Du J, Yang F, Zhang S, Jin Q. Deciphering the bat virome catalog to better understand the ecological diversity of bat viruses and the bat origin of emerging infectious diseases. ISME J. 2016;10:609-20.

62. Kluge M, Campos FS, Tavares M, de Amorim DB, Valdez FP, Giongo A, Roehe PM, Franco AC. Metagenomic survey of viral diversity obtained from feces of Subantarctic and South American fur seals. PLoS One. 2016;11:e0151921.

63. Van Ranst M, Fuse A, Fiten P, Beuken E, Pfister H, Burk RD, Opdenakker G. Human papillomavirus type 13 and pygmy chimpanzee papillomavirus type 1: comparison of the genome organizations. Virology. 1992;190:587-96.

64. Tachezy R, Duson G, Rector A, Jenson AB, Sundberg JP, Van Ranst M. Cloning and genomic characterization of Felis domesticus papillomavirus type 1. Virology. 2002;301:313-21.

65. Brandt S, Schoster A, Tober R, Kainzbauer C, Burgstaller JP, Haralambus R, Steinborn R, Hinterhofer C, Stanek C. Consistent detection of bovine papillomavirus in lesions, intact skin and peripheral blood mononuclear cells of horses affected by hoof canker. Equine Vet J. 2011;43:202-9.

66. Ng TFF, Miller MA, Kondov NO, Dodd EM, Batac F, Manzer M, Ives S, Saliki JT, Deng X, Delwart E. Oral papillomatosis caused by Enhydra lutris papillomavirus 1 (EIPV-1) in southern sea otters (Enhydra lutris nereis) in California, USA. J Wildl Dis. 2015;51:446-53.

67. Rector A, Van Doorslaer K, Bertelsen M, Barker IK, Olberg R-A, Lemey P, Sundberg JP, Van Ranst M. Isolation and cloning of the raccoon (Procyon lotor) papillomavirus type 1 by using degenerate papillomavirus-specific primers. J Gen Virol. 2005;86(Pt 7):2029-33. 
68. Koonin EV, Wolf YI, Nagasaki K, Dolja W. The Big Bang of picorna-like virus evolution antedates the radiation of eukaryotic supergroups. Nat Rev Microbiol. 2008;6:925-39.

69. Sikorski A, Massaro M, Kraberger S, Young LM, Smalley D, Martin DP, Varsani A. Novel myco-like DNA viruses discovered in the faecal matter of various animals. Virus Res. 2013;177:209-16.

70. Biagini P. Classification of TTV and related viruses (anelloviruses). Curr Top Microbiol Immunol. 2009;331:21-33.

71. de Villiers E-M, Borkosky SS, Kimmel R, Gunst K, Fei J-W. The diversity of torque teno viruses: in vitro replication leads to the formation of additional replication-competent subviral molecules. J Virol. 2011:85:7284-95.

72. Nishiyama S, Dutia BM, Stewart JP, Meredith AL, Shaw DJ, Simmonds P, Sharp CP. Identification of novel anelloviruses with broad diversity in UK rodents. J Gen Virol. 2014;95(Pt 7):1544-53.

73. Ninomiya M, Takahashi M, Nishizawa T, Shimosegawa T, Okamoto H. Development of PCR assays with nested primers specific for differential detection of three human anelloviruses and early acquisition of dual or triple infection during infancy. J Clin Microbiol. 2008;46:507-14.

74. Vasconcelos HCF, Cataldo M, Niel C. Mixed infections of adults and children with multiple TTV-like mini virus isolates. J Med Virol. 2002;68:291-8.

75. Okamoto H, Takahashi M, Nishizawa T, Ukita M, Fukuda M, Tsuda F, Miyakawa Y, Mayumi M. Marked genomic heterogeneity and frequent mixed infection of $\Pi T$ virus demonstrated by PCR with primers from coding and noncoding regions. Virology. 1999;259:428-36.

76. Kapusinszky B, Mulvaney U, Jasinska AJ, Deng X, Freimer N, Delwart E. Local virus extinctions following a host population bottleneck. J Virol. 2015;89:8152-61.

77. Béland K, Dore-Nguyen M, Gagné M-J, Patey N, Brassard J, Alvarez F, Halac U. Torque Teno virus load as a biomarker of immunosuppression? New hopes and insights. J Infect Dis. 2014;210:668-70.

78. Maggi F, Pifferi M, Michelucci A, Albani M, Sbranti S, Lanini L, Simi P, Macchia P, Pistello M, Bendinelli M. Torque teno virus viremia load size in patients with selected congenital defects of innate immunity. Clin Vaccine Immunol. 2011;18:692-4.

79. Virgin HW. The Virome in mammalian physiology and disease. Cell. 2014;157:142-50.

\section{Submit your next manuscript to BioMed Central and we will help you at every step:}

- We accept pre-submission inquiries

- Our selector tool helps you to find the most relevant journal

- We provide round the clock customer support

- Convenient online submission

- Thorough peer review

- Inclusion in PubMed and all major indexing services

- Maximum visibility for your research

Submit your manuscript at www.biomedcentral.com/submit

) Biomed Central 